Liu, Y. (2016) On the space-time correlation of heat release rate in turbulent premixed flames.

Combust. Sci. Technol. 188 (2), 166-189. DOI:10.1080/00102202.2015.1085861

\title{
On the space-time correlation of heat release rate in turbulent premixed flames
}

\author{
Y. Liu \\ Department of Mechanical Engineering Sciences, University of Surrey, \\ Guildford GU2 7XH, United Kingdom
}

\begin{abstract}
The spectral characteristics of combustion noise is dictated by the temporal correlation of the heat release rate fluctuation. This paper investigates the two-point space-time correlation of heat release rate fluctuations by analysing recent direct numerical simulation (DNS) data of turbulent premixed flames. The results show that this correlation decays rapidly in both space and time due to the abrupt fluctuation of the heat release rate. The two-point space-time correlation can be modelled by Gaussian-type functions including the streamwise direction with strong convection. A flat low-frequency dependence is observed for the spectrum of the correlation function, which will allow the modelling of the spectral peak and the low-frequency spectral slope of combustion noise when multiplying the term $\omega^{2}$ ( $\omega$ is the angular frequency). This exploratory work shows the potential of this correlation function for capturing the spectral shape of combustion noise.

Keywords: two-point space-time correlation, correlation volume and length scale, combustion noise spectrum, heat release rate fluctuation, turbulent premixed flames.
\end{abstract}

Running title: Space-time heat release rate correlation

\section{Introduction}

Sound emission is produced during turbulent combustion processes because they are inherently unsteady. Over the last decade the noise from unsteady turbulent combustion is emerging as an important noise source in particular for lean-burn gas turbines that generally involve highly unsteady flames (Swaminathan et al., 2011,a). Since lean burning is the potential way forward for environmentally friendly engines due to its great advantage of very low NOx emission, this noise source from turbulent combustion needs to be addressed. Therefore, the source mechanisms of combustion noise needs to be understood thoroughly. This paper considers only direct sound generation from open, turbulent premixed flames. The major source of direct combustion noise has been identified as the monopole source (e.g. Hurle et al., 1968; Strahle, 1978; Jones, 1979; Crighton et al., 1992; Swaminathan et al., 2011a; Liu, 2015) arising from the fluctuation of heat release rate which causes unsteady expansion of the turbulent flame volume. Although flame annihilation events produce significant noise as a result of rapid destructions of flame surface area and hence heat release when two flame surfaces interact (Talei et al., 2011, 2012, 2013), it is out of the scope of this study.

Combustion noise has been studied extensively with many research efforts on the overall sound power (e.g. Roberts and Leventhall, 1973; Strahle and Shivashankara, 
1975; Strahle, 1978; Putnam, 1976; Kilham and Kirmani, 1979; Swaminathan et al., 2011,a; Liu et al., 2012). However, the source mechanisms for combustion noise are still less understood compared with aerodynamic noise (for example, jet noise), and its prediction remains a challenging issue. Theoretical modelling of the overall sound pressure level (OASPL) of combustion noise in unbounded space (Crighton et al., 1992; Bragg, 1963; Strahle, 1971; Kotake, 1975; Clavin and Siggia, 1991) requires understanding the correlation between spatially distributed heat release rate oscillations as first recognised by Kotake (1975), namely the two-point cross-correlation of the rate of change of the heat release rate fluctuation. This two-point correlation along with the associated correlation volume $v_{\text {cor }}$ is central to the prediction of the OASPL of combustion noise. However, this correlation was not investigated sufficiently despite many empirical length scale of $v_{\text {cor }}$ proposed in previous studies (e.g. laminar flame thickness $\delta_{L}$ (Bragg, 1963), turbulence integral length scale (Strahle, 1971), turbulent flame-brush thickness (Wäsle et al., 2005; Hirsch et al., 2007)), and it is still unclear about the appropriate length scale for $v_{\text {cor }}$. Recently, Swaminathan et al. $(2011, \mathrm{a})$ modelled this correlation through direct numerical simulation (DNS) (Rutland and Cant, 1994; Nada et al., 2004, 2005) and laser diagnostics (Balachandran et al., 2005) data of turbulent premixed flames, and estimated the correlation volume as $\delta_{L}^{3} / 8$ by assuming isotropic correlation, where $\delta_{L}$ is the laminar flame thermal thickness. Liu et al. (2012) revisited the two-point correlation by analysing recent DNS data of turbulent premixed V-flames (Dunstan et al., 2011) and obtained a larger value of $0.2 \delta_{L}^{3}$ because the correlation was observed to be anisotropic in different directions.

In contrast to the overall sound emissions from turbulent premixed flames, the attention received to their spectral characteristics is far from sufficient in literature (Kotake and Takamoto, 1987, 1990; Rajaram and Lieuwen, 2003, 2009; Rajaram, 2007; Liu et al., 2012a, 2014; Liu, 2015, for example). The limited experimental studies have shown that the spectrum of combustion noise is broadband in nature with a peak in the spectrum at $f=f_{\text {peak }}$ and a low-frequency dependence $f^{\beta}$ where the slope $\beta$ was found to be nearly a constant $\beta \approx 2$ in a recent systematic experimental study (Rajaram, 2007; Rajaram and Lieuwen, 2009). Modelling the spectral characteristics of turbulent combustion noise is very challenging due to the highly nonlinear relationship between the unsteady heat release and the underlying turbulent velocity fluctuations, and this requires knowledge on the temporal correlation of heat release rate fluctuations. In recent studies on the two-point spatial correlation of the rate of change of heat release rate fluctuations in order to predict the far-field OASPL (Swaminathan et al., 2011,a; Liu et al., 2012), in particular that of Liu et al. (2012) using the same V-flame DNS data (Dunstan et al., 2011) as in the present study, no attempts were made to address the correlation in time and hence no information was available to study the spectral features of combustion noise.

Therefore, the present work will focus on the temporal correlation of heat release rate fluctuations produced during turbulent combustion which can be applied to the analytical modelling of combustion noise spectrum. One kind of temporal correlation of heat release rate fluctuations is the two-point space-time correlation which has been successfully applied to model the spectrum of fluid dynamic noise in non-reacting flows, for example jet noise spectrum (e.g. Karabasov et al., 2010, 2011; Depuru Mohan 
et al., 2015). However, the two-point space-time correlation of the heat release rate fluctuation in turbulent flames has scarcely been reported in prior literature (Liu et al., 2011) primarily because reliable heat release rate data are absent in previous numerical simulations or measurements. Recently, thanks to the advancement in computing technologies and laser metrology, high-fidelity data of heat release rate fluctuations have made it feasible to analyse this temporal correlation directly. Hemchandra and Lieuwen (2010) estimated numerically the space-time correlation of local burning area fluctuations, which are intimately related to the heat release rate fluctuations at the local flame surface, for a turbulent premixed V-flame, but did not consider directly the space-time correlation of heat release rate fluctuations inside the flame brush. Moreover, the correlation volume and its length scale associated with the two-point space-time correlation function must be time dependent (see Section 4.6), and it is therefore of interest to explore the frequency dependence of these spatial scales which has not been addressed yet to the best of the author's knowledge.

The primary objectives of this paper are: (i) to investigate the characteristics of the two-point space-time correlation of the heat release rate fluctuations through analysing recent V-flame DNS data (Dunstan et al., 2011); (ii) to develop a theoretical model for the two-point space-time correlation function from the DNS results; and (iii) to study the length and volume scales of this correlation and their frequency dependence. The remaining paper is organised as follows. In Section 2, the two-point space-time correlation and its relation to the combustion noise spectrum are introduced briefly. The salient features of the V-flame DNS data and the analyses are presented in Section 3. The results of the two-point space-time correlation, including the correlation function and its model along with the integral length scales and correlation volume, are discussed in Section 4. The conclusions and suggestions for further work are summarised in Section 5 .

\section{Theoretical modelling}

\subsection{Combustion noise spectrum}

Based upon the foundation laid by Lighthill's theory (Lighthill, 1952, 1954), Dowling (in Crighton et al., 1992) has derived explicitly the sound field radiated from a turbulent reacting flow. The dominant source in most cases, e.g. in open flames where low Mach number conditions apply, is the direct noise from unsteady heat input. Therefore, the governing linear wave equation can be expressed in a reduced form as:

$$
\frac{1}{a_{0}^{2}} \frac{\partial^{2} p^{\prime}}{\partial t^{2}}-\nabla^{2} p^{\prime}=\frac{\partial}{\partial t}\left[\frac{\rho_{0}(\gamma-1)}{\rho a^{2}} \dot{Q}^{\prime}(\boldsymbol{y}, t)\right],
$$

where $p^{\prime}$ is the acoustic pressure, $\gamma$ is the specific heat ratio, and $\dot{Q}^{\prime}$ is the heat release rate fluctuation per unit volume; $\rho$ and $a$ denote the air density and speed of sound within the flame which are different from $\rho_{0}$ and $a_{0}$ in ambient air. By assuming that the combustion occurs at ambient pressure and $\gamma$ is independent of temperature, one has $\rho a^{2}=\gamma p_{0}=\rho_{0} a_{0}^{2}$ and thus the wave equation (1) simplifies to

$$
\frac{1}{a_{0}^{2}} \frac{\partial^{2} p^{\prime}}{\partial t^{2}}-\nabla^{2} p^{\prime}=\frac{(\gamma-1)}{a_{0}^{2}} \frac{\partial \dot{Q}^{\prime}(\boldsymbol{y}, t)}{\partial t} .
$$


For simplicity, in Eq. (2) the effects of convection due to mean flow (Dowling, 1979) and refraction of sound due to temperature inhomogeneities (Strahle, 1973) are ignored.

For an open flame, the formal solution of Eq. (2) is expressed as:

$$
p^{\prime}(\boldsymbol{x}, t)=\frac{(\gamma-1)}{4 \pi r a_{0}^{2}} \frac{\partial}{\partial t} \int_{v_{f}} \dot{Q}^{\prime}\left(\boldsymbol{y}, t-r / a_{0}\right) \mathrm{d}^{3} \boldsymbol{y}
$$

by using the Green's function in free space, where $\boldsymbol{x}$ is an observer position in far field, $\boldsymbol{y}$ denotes the source position within the flame brush $v_{f}$ which is assumed to be acoustically compact; hence the observer distance is approximated as $r \approx|\boldsymbol{x}|$ from an origin in $v_{f}$. The auto-correlation function of a stochastic signal of sound pressure perturbation $p^{\prime}(\boldsymbol{x}, t)$ is defined as (Dowling and Ffowcs Williams, 1983)

$$
P(\boldsymbol{x}, \tau)=\frac{(\gamma-1)^{2}}{16 \pi^{2} r^{2} a_{0}^{4}} \frac{\partial^{2}}{\partial t^{2}} \int_{v_{f}} \int_{v_{\mathrm{cor}}} \overline{\dot{Q}^{\prime}(\boldsymbol{y}, t) \dot{Q}^{\prime}(\boldsymbol{y}+\boldsymbol{\Delta}, t+\tau)} \mathrm{d}^{3} \boldsymbol{\Delta} \mathrm{d}^{3} \boldsymbol{y}
$$

where the overbar refers to an averaging process over time and location, $\boldsymbol{\Delta}$ is the separation vector between two source positions within the flame brush, and $\tau$ is the retarded time lag between noise radiated from the two locations. The correlation volume $v_{\text {cor }}$ originates from Bragg's theory (Bragg, 1963) and represents the volume over which $\dot{Q}^{\prime}$ is correlated.

The frequency spectrum of the pressure perturbation $p^{\prime}$ is obtained by applying a Fourier transform (FT) of Eq. (3),

$$
\hat{p}(\boldsymbol{x}, \omega)=\frac{(\gamma-1)}{4 \pi r a_{0}^{2}} i \omega \int_{v_{f}} \hat{\dot{Q}}(\boldsymbol{y}, \omega) e^{-\mathrm{i} k_{0} r} \mathrm{~d}^{3} \boldsymbol{y}
$$

where $\omega$ is the angular frequency and the wavenumber $k_{0}=\omega / a_{0}$. Similarly, the power spectral density (PSD) $\hat{P}(\boldsymbol{x}, \omega)$, a measurable quantity in experiments, can be derived in a general form as

$$
\hat{P}(\boldsymbol{x}, \omega)=\frac{(\gamma-1)^{2} \omega^{2}}{32 \pi^{3} r^{2} a_{0}^{4}} \int_{v_{f}} \int_{v_{\text {cor }}} \int_{-\infty}^{\infty} \overline{\hat{Q}}(\boldsymbol{y}, \omega) \hat{\dot{Q}}\left(\boldsymbol{y}+\boldsymbol{\Delta}, \omega^{\prime}\right) \mathrm{d} \omega^{\prime} \mathrm{d}^{3} \boldsymbol{\Delta} \mathrm{d}^{3} \boldsymbol{y}
$$

after an FT of Eq. (4) and using the relationship between the PSD and the FT of the signal $p^{\prime}(\boldsymbol{x}, t)$ (see Dowling and Ffowcs Williams, 1983, for example), where $\omega^{\prime}$ is a dummy variable. Noting that the double time derivative in Eq. (4) is simply replaced by the term $\omega^{2}$ during the Fourier transform, hereafter we will consider the space-time correlation of the heat release rate fluctuation instead of the rate of change of $\dot{Q}^{\prime}$.

\subsection{Two-point space-time correlation}

The two-point space-time correlation has been commonly used in modelling fluid dynamic noise in non-reacting flows (Karabasov et al., 2010, for example). In the case of noise generation from turbulent combustion, the two-point space-time correlation of the fluctuating heat release rate, defined as $\overline{\dot{Q}^{\prime}(\boldsymbol{y}, t) \dot{Q}^{\prime}(\boldsymbol{y}+\boldsymbol{\Delta}, t+\tau)}$ in Eq. (4), indicates that the source mechanism of combustion noise is the cross-correlation of the heat release rate fluctuation $\dot{Q}^{\prime}(\boldsymbol{y}, t)$ distributed over the flame brush and that its spectral content is controlled by the temporal correlation of $\dot{Q}^{\prime}$. 
The common practices of analysing turbulent premixed flames (e.g. Swaminathan et al., 2011,a; Liu et al., 2012,a; Liu and Echekki, 2015) suggest to use a progress variable $c$ defined by fuel mass fraction (Poinsot and Veynante, 2005). The progress variable $c$ takes the value of zero in reactants and unity in products. In doing so, the heat release rate $\dot{Q}$ and chemical reaction rate $\dot{w}$ are related as $\dot{Q}=Y_{f, u} H \dot{w}$, where the fuel mass fraction in unburnt reactants, $Y_{f, u}$, is uniform in premixed flames, and $H$ is the lower heating value of the fuel (a table constant in unit of $\mathrm{J} \mathrm{kg}^{-1}$ ). This is a good approximation even using a complex chemical kinetics to model combustion (Poinsot and Veynante, 2005). The heat release rate $\dot{Q}$ is then simply replaced by the reaction rate $\dot{w}$ in subsequent analysis, and the two-point space-time correlation of $\dot{Q}^{\prime}$ in Eq. (4) can be expressed as

$$
\begin{aligned}
& \overline{\dot{Q}^{\prime}(\boldsymbol{y}, t) \dot{Q}^{\prime}(\boldsymbol{y}+\boldsymbol{\Delta}, t+\tau)}=Y_{f, u}^{2} H^{2} \overline{\dot{w}^{\prime}(\boldsymbol{y}, t) \dot{w}^{\prime}(\boldsymbol{y}+\boldsymbol{\Delta}, t+\tau)}, \\
& \overline{\dot{w}^{\prime}(\boldsymbol{y}, t) \dot{w}^{\prime}(\boldsymbol{y}+\boldsymbol{\Delta}, t+\tau)}=\Omega(\boldsymbol{\Delta}, \tau) \overline{\dot{w}^{\prime 2}(\boldsymbol{y}, t)}
\end{aligned}
$$

where $\Omega(\boldsymbol{\Delta}, \tau)$ is the two-point space-time correlation function for $\dot{Q}^{\prime}$, and all terms with overbar are averaged over time $t$. It is obvious in Eq. (7b) that the correlation function $\Omega$ depends on the source position $\boldsymbol{y}$ in $v_{f}$ as well as the spatial separation $\boldsymbol{\Delta}$ and time lag $\tau$. However, as will be shown in Section 4.2, the dependence on $\boldsymbol{y}$ is nearly negligible and hence the correlation function can be expressed as $\Omega(\boldsymbol{\Delta}, \tau)$. A detailed discussion on the assumptions of Eqs. (7a) and (7b) has been given in Swaminathan et al. (2011a).

Similar to the PSD of $\hat{P}(\boldsymbol{x}, \omega)$, the frequency spectrum of the correlation function $\Omega(\boldsymbol{\Delta}, \tau)$ in time domain can be obtained by applying an FT to Eq. (7),

$$
\frac{1}{2 \pi} \int_{-\infty}^{\infty} \overline{\hat{\dot{Q}}(\boldsymbol{y}, \omega) \hat{\dot{Q}}\left(\boldsymbol{y}+\boldsymbol{\Delta}, \omega^{\prime}\right)} \mathrm{d} \omega^{\prime}=Y_{f, u}^{2} H^{2} \hat{\Omega}(\boldsymbol{\Delta}, \omega) \overline{\dot{w}^{\prime 2}(\boldsymbol{y}, t)} .
$$

Substituting Eq. (8) into Eq. (6), the spectrum of the far-field PSD of the form can be expressed in terms of the two-point space-time correlation:

$$
\hat{P}(\boldsymbol{x}, \omega)=\frac{(\gamma-1)^{2}}{16 \pi^{2} r^{2} a_{0}^{4}} Y_{f, u}^{2} H^{2} \omega^{2} \int_{v_{f}} \overline{\dot{w}^{\prime 2}(\boldsymbol{y}, t)} \mathrm{d}^{3} \boldsymbol{y} \int_{v_{\mathrm{cor}}} \hat{\Omega}(\boldsymbol{\Delta}, \omega) \mathrm{d}^{3} \boldsymbol{\Delta},
$$

where the double integral in Eq. (6) is divided into two single integrals over the flame brush $v_{f}$ and correlation volume $v_{\text {cor }}$, respectively. The terms $\overline{\dot{w}^{\prime 2}}$ and $v_{f}$ contained in the first integral are turbulence-thermo-chemistry dependent, and the influences of turbulence are manifested through the two terms (Swaminathan et al., 2011a). The second integral over the correlation volume is independent of the flame brush since the correlation function $\Omega$ does not depend on source positions inside $v_{f}$ (see Section 4.2). As noted by Swaminathan et al. (2011a), the correlation function and the associated correlation volume $v_{\text {cor }}$ are dominated by thermochemical processes; the effect of turbulence on $\Omega$ is negligibly small and is hence ignored in the analysis. In the following the DNS data of turbulent premixed V-flames (Dunstan et al., 2011) will be employed to analyse the correlation function $\Omega(\boldsymbol{\Delta}, \tau)$ and its spectrum.

\section{$3 \quad$ V-flame DNS data}

One of the canonical turbulent flame configurations is the single V-flame. Extensive experimental (e.g. Smith and Gouldin, 1978; Veynante et al., 1994; Gouldin, 1996; 
Shepherd, 1996; Sattler et al., 2002) and numerical (e.g. Alshaalan and Rutland, 1998; Domingo et al., 2005; Bell et al., 2005) studies on this type of flame have been carried out previously. Recently, Dunstan et al. (2011) reported 3D fully compressible DNS of premixed turbulent V-flames to evaluate the turbulent flame speed. A cubic computational domain was used and Navier-Stokes Characteristic Boundary Conditions (NSCBC) (Poinsot and Lele, 1992) were applied in the streamwise $x$-direction and the transverse $y$-direction. They modified the standard NSCBC to accommodate the steep thermal and compositional gradients as a result of the interaction of the flame front with the outlet of the computation domain. Periodic conditions were specified in the spanwise $z$-direction in which the flame holder is aligned. The flame holder stabilises the flame by applying species mass fractions and velocities through a Gaussian weighting function over it. This V-flame DNS has been used in an earlier study (Liu et al., 2012) to investigate the two-point spatial correlation of the rate of change of heat release rate fluctuations. Section 3.3 will introduce some general features of the V-flame and complete details of this V-flame DNS can be found in Dunstan et al. (2011).

\subsection{Flame conditions}

Table 1: Parameters of flame conditions for the DNS V-flames (Dunstan et al., 2011).

\begin{tabular}{cccccccc}
\hline Case & $u_{\mathrm{rms}}^{+}$ & $\bar{u}^{+}$ & $\Lambda^{+}$ & $\mathrm{Re}$ & $\mathrm{Da}$ & $\tau_{D}^{+}$ & $\Delta t(\mathrm{~s})$ \\
\hline III & 6.0 & 24.86 & 3.43 & 92 & 0.57 & 1.19 & $1 \times 10^{-5}$ \\
\hline
\end{tabular}

Dunstan et al. (2011) considered three V-flame cases with weak, moderate and high intensity turbulence. In this study the case of high intensity turbulence, Case III, is used to study the two-point space-time correlation because the faster movement of the flame front in this case is expected to produce better convergence of the correlation in time. The typical flame conditions of Case III are summarised in Table 1. The chemical mechanism for preheated reactants was assumed to be single step and unity Lewis numbers were used in all cases. The thermochemical parameters were specified as: laminar flame thermal thickness $\delta_{L}=0.43 \mathrm{~mm}$, flame speed $S_{L}=0.6 \mathrm{~m} \mathrm{~s}^{-1}$, and laminar flame time $\tau_{L}=\delta_{L} / S_{L}=0.71 \mathrm{~ms}$, which represent a premixed methane-air flame with an equivalence ratio of $\phi \approx 0.6$. In Table 1 , the superscript + denotes a normalisation using the laminar flame thickness $\delta_{L}$, flame speed $S_{L}$ and the unburnt reactant density $\rho_{u}$. Most of the parameters in Table 1 are measured at the inlet of the computational domain: turbulence intensity $u_{\text {rms }}$ (i.e. the root-mean-square of turbulence velocity fluctuation), mean streamwise velocity $\bar{u}$, turbulence integral length scale $\Lambda$, turbulence Reynolds number Re based on $u_{\text {rms }}$ and $\Lambda$, and Damköhler number $\mathrm{Da}=\Lambda^{+} / u_{\mathrm{rms}}^{+}$.

The computational domain has a spatial resolution of 512 uniform grid points in each direction over a length of $12.77 \mathrm{~mm}\left(29.7 \delta_{L}\right)$, resulting in more than 17 grid points inside one laminar flame thickness. A distance of $3.48 \delta_{L}$ was set between the flame holder and the inlet of the computational domain to ensure that stable combustion was achieved and the flame holder effect on the flame statistics downstream was minimised. 
The sample fields were collected at positions at least one $\delta_{L}$ away from the boundaries of the computational domain to eliminate any possible boundary influences. After a fully developed turbulence state has been reached and one flow-through time $\tau_{D}$ (i.e. the mean convection time from the inlet to outlet boundaries) had been run to ensure the decay of initial transients (Dunstan et al., 2011), data were collected consecutively during one time length of $\tau_{D}$ at positions at least one $\delta_{L}$ away from any boundaries of the computational domain to avoid any possible influences of boundary conditions. With regard to the temporal resolution, 85 time steps $\left(\Delta t=10^{-5} \mathrm{~s}\right)$ were taken for Case III. The time length of the Case III data $\left(\tau_{D}^{+}=1.19\right)$ is sufficient for constructing the two-point space-time correlation as it decays rapidly in time (see Section 4.3).

\subsection{Data processing}

The construction of the correlation function $\Omega(\boldsymbol{\Delta}, \tau)$ requires the reaction rate fluctuations $\dot{w}^{\prime}$ which are calculated by subtracting the mean reaction rate $\bar{w}$ from the instantaneous reaction rate $\dot{w}$. First, the averaging process is applied to obtain the mean reaction rate (Liu et al., 2012),

$$
\overline{\dot{w}}(x, y)=\frac{1}{N_{t} N_{z}} \sum_{n=1}^{N t} \sum_{k=1}^{N_{z}} \dot{w}\left(x, y ; t_{n}, z_{k}\right),
$$

where $N_{t}=85$ is the number of sample fields collected over one flow-through time, and $N_{z}=512$ is the number of grid points in the periodic spanwise direction. The averaging in Eq. (10) improves the statistical convergence. The reaction rate fluctuation is obtained by calculating $\dot{w}^{\prime}=\dot{w}-\overline{\dot{w}}$ on point-by-point basis in the $\dot{w}$ fields saved at the $N_{t}$ time steps. The construction of the correlation function $\Omega$ is then straightforward following Eq. (7b). Note that when constructing a temporal correlation, the effective time length of $\tau$ for the correlation function is reduced to

$$
\tau_{e}=\left(N_{t}-N_{\text {ave }}\right) \cdot \Delta t
$$

where $N_{\text {ave }}$ is the number of sample fields required to ensure sufficient accuracy during the time averaging. In the present study, $N_{\text {ave }}=42$ is taken resulting in an effective time length of $\tau_{e}^{+}=0.6$. This effective time length is sufficient for the correlation function since $\Omega\left(\tau^{+}\right)$reaches a small value of 0.05 around $\tau^{+}=0.1$, as will be shown in Figure 5 .

The two-point space-time correlation function $\Omega(\boldsymbol{\Delta}, \tau)$ is analysed at selected local sample points inside the flame brush. Figure 1 illustrates the two-dimensional (2D) contours of the Favre-averaged (i.e. density weighted) progress variable $\tilde{c}$. The progress variable $\tilde{c}$ can be regarded as dependent only on the $(x, y)$ plane because of the predominantly 2D mean flow field of the DNS flame. Hence for a given streamwise location $x$ inside the flame brush, the transverse position $y$ can be simply denoted by $\tilde{c}$. The sample points for $\Omega(\Delta, \tau)$ are restricted in the range $0.1<\tilde{c}<0.9$ to ensure that regions with $\overline{\dot{w}}$ close to zero are avoided for meaningful statistics (Swaminathan et al., 2011,a). Three streamwise sample positions, $x_{o}^{+}=16.7,20.4$ and 24.2 as in Dunstan et al. (2011), are selected (see Figure 1) and for each position of $x_{o}^{+}$a series of $\tilde{c}$ values, $0.2,0.3, \ldots, 0.8$, are taken along the $y$-direction for each flame branch. The correlation function $\Omega$ is then evaluated by taking the separation distances $\Delta_{x}, \Delta_{y}$ and $\Delta_{z}$ from 


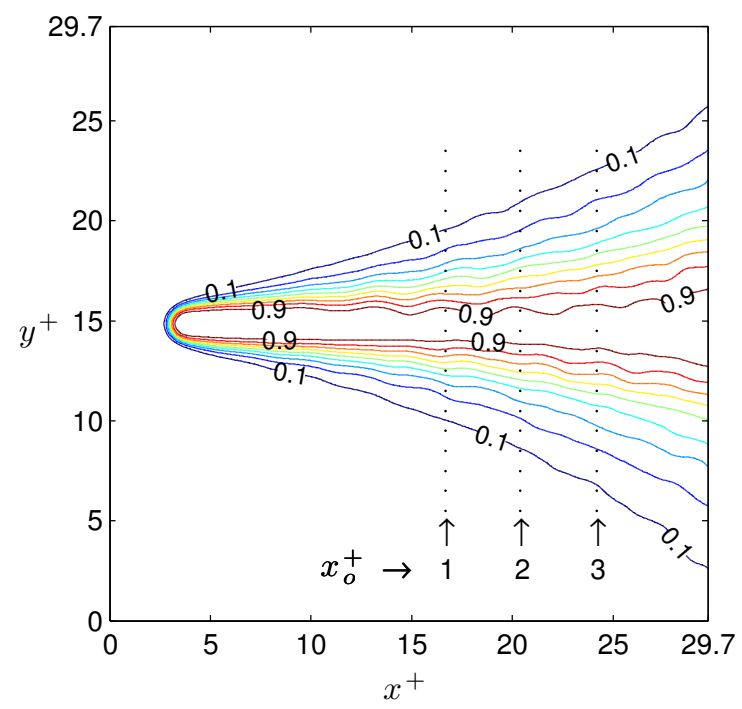

Figure 1: Contours of $\tilde{c}$ in the $\left(x^{+}, y^{+}\right)$plane of the DNS V-flame along with the three streamwise sample positions located at $x_{o}^{+}=16.7,20.4$ and 24.2 .

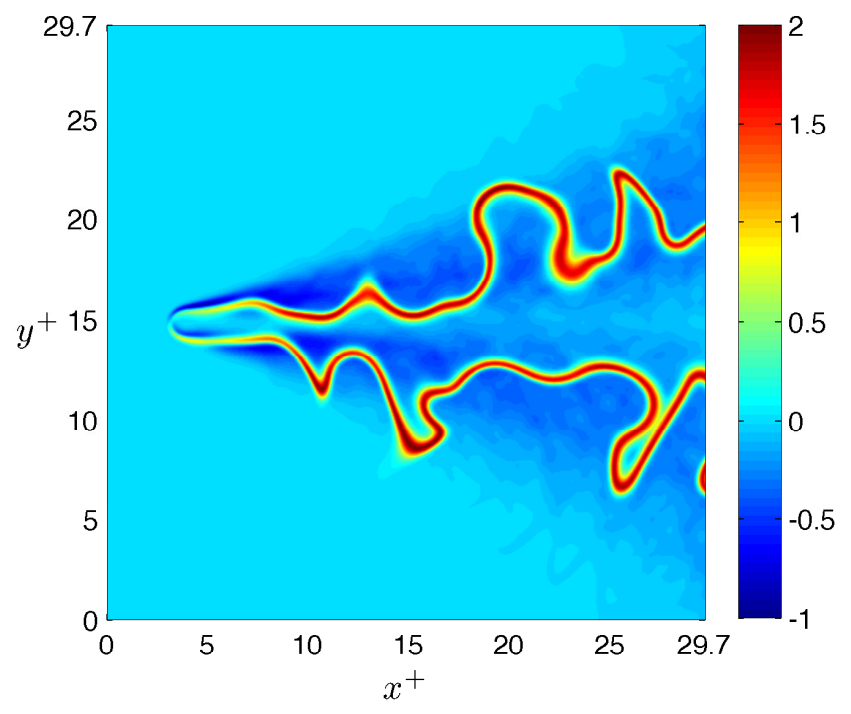

Figure 2: Typical contours of the instantaneous fluctuating reaction rate $\dot{w}^{\prime+}$ (normalised by $\left.\rho_{u} S_{L} / \delta_{L}\right)$ in the mid $\left(x^{+}, y^{+}\right.$) plane of the DNS V-flame.

these sample positions and finally averaging between the two flame branches (Liu et al., 2012).

\subsection{Flame features}

Figure 1 displays the contour plot of $\tilde{c}$ in the $\left(x^{+}, y^{+}\right)$plane for Case III. Also shown in Figure 1 are the three streamwise sample positions located at $x_{o}^{+}=16.7,20.4$ and 24.2. A slightly curved mean flame can be seen as in a typical V-flame due to the increase of the orientation angle of the mean flame brush to the reactant stream along the streamwise $x$-direction. The size of the flame brush $v_{f}$ can be roughly inferred from 
the distribution of the $\tilde{c}$ contours. Figure 1 also shows the effects of the high turbulence intensity $u_{\mathrm{rms}}^{+}$and mean velocity $\bar{u}^{+}$in terms of the enlarged turbulent flame brush and the reduced distance between the flame branches (Dunstan et al., 2011), which is in agreement with observations in previous studies (for example Lipatnikov and Chomaik, 2002).

Figure 2 illustrates typical contours of the instantaneous fluctuating reaction rate $\dot{w}^{\prime+}$ normalised by $\rho_{u} S_{L} / \delta_{L}$ in the mid $\left(x^{+}, y^{+}\right)$plane. The thin regions of the $\dot{w}^{\prime}$ contours indicate a typical thickness of the reaction zone, $\delta_{\dot{w}} \approx \delta_{L}$, which remains almost one laminar flame thickness along the flame front (Liu et al., 2012). The value of $\delta_{\dot{w}}$ can increase up to $2 \delta_{L}$ in regions where the curvature of the flame front becomes large. The influence of the high turbulence intensity is also manifested in the sinuous contours with large curvature. Note that although the positive values of $\dot{w}^{\prime}$ are evident within the thin reaction zone, there are negative reaction rate fluctuations distributed over the adjacent areas mostly within the flame brush.

Figure 3 shows the fluctuating reaction rate $\dot{w}^{\prime+}\left(t^{+}\right)$at the three streamwise positions and four transverse positions for the lower flame branch. As the flame front sweeps past a sample point, $\dot{w}^{\prime}$ rises sharply and then decreases to a negative value. This negative $\dot{w}^{\prime}$ will remain for a period of time after the flame front has past and the local

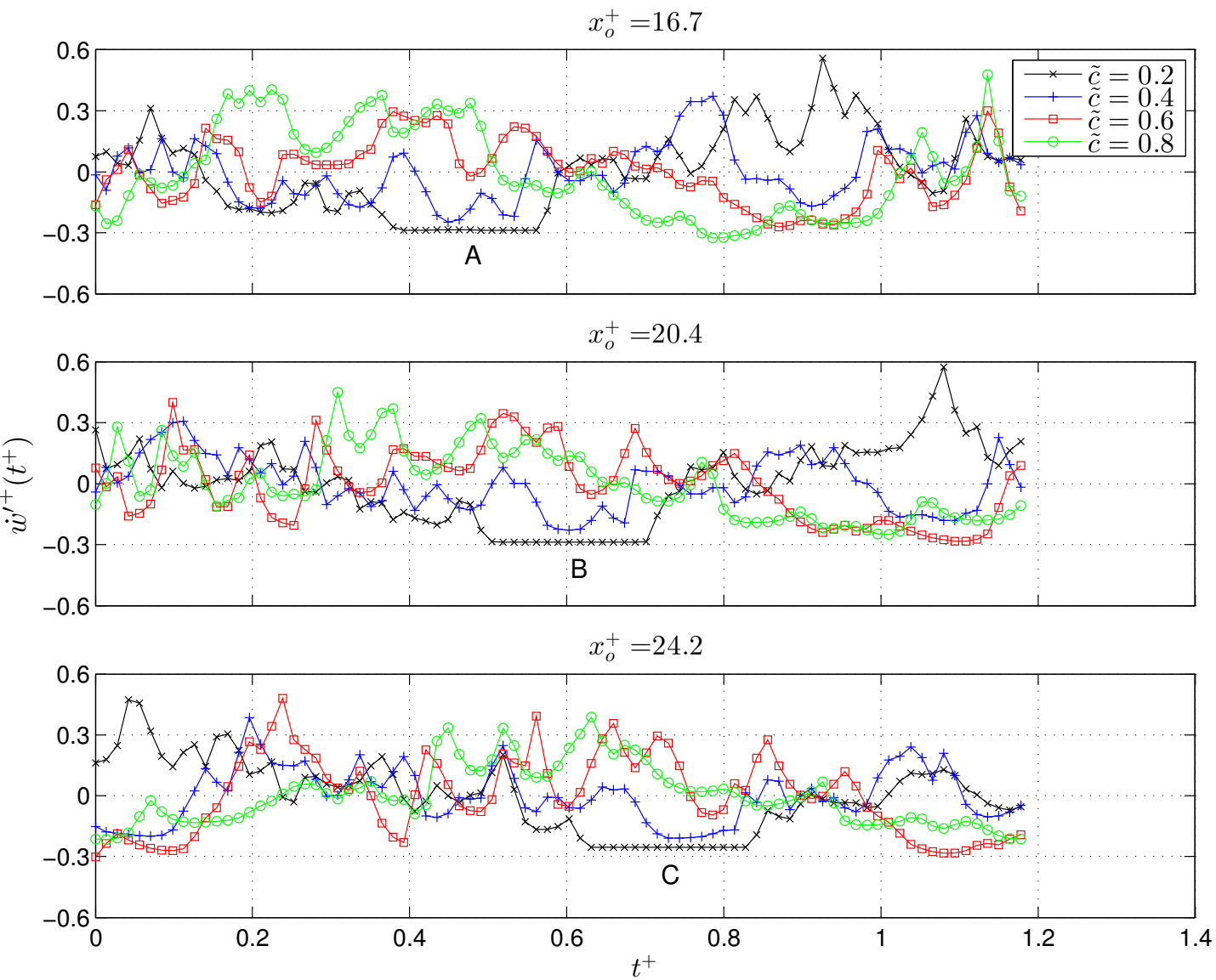

Figure 3: Time traces of the local reaction rate fluctuations $\dot{w}^{\prime+}$ (normalised by $\left.\rho_{u} S_{L} / \delta_{L}\right)$ at various positions in the lower flame branch. 
reaction has finished, resulting in a period of time trace with nearly flat amplitude. This flat time segment is seen more obviously at positions close to the flame brush boundary (i.e. $\tilde{c}=0.2$ ) due to the relatively long sweeping interval at these positions. A convection effect is indicated by the flat time segments at the three $x_{o}^{+}$positions that appear in succession as the flame front translates downstream with the mean flow (for example, the segments at $\tilde{c}=0.2$, marked by A, B and C in Figure 3 ).

\section{Results and discussion}

\subsection{Correlation model}

The two-point space-time correlation of the fluctuating reaction rate $\dot{w}^{\prime}(\boldsymbol{y}, t)$ is constructed at the selected sample points following the definition in Eq. (7b) and the processing procedures described in Section 3.2. A theoretical model for the correlation function $\Omega(\boldsymbol{\Delta}, \tau)$ can be developed by analysing the V-flame DNS data (Dunstan et al., 2011). In a series of recent studies on modelling the two-point space-time correlation of jet noise sources (e.g. Karabasov et al., 2010, 2011; Depuru Mohan et al., 2015), it was observed that this correlation can be well represented by the Gaussian-type function commonly used in classical turbulence. Tam (2015) recently proposed that the spectral shape of combustion noise from hydrocarbon fuels is the same as the similarity spectrum of the large turbulence structure noise from high-speed jets, regardless of the turbulence level or fuel properties. Since the spectral characteristics of combustion noise are dictated by the temporal correlation of heat release rate fluctuations, it is natural to adopt the Gaussian function to model this correlation of combustion noise sources. In addition, the Gaussian function was also found to well approximate the two-point spatial correlation of heat release rate fluctuations using DNS and laser diagnostics data (Swaminathan et al., 2011,a; Liu et al., 2012).

Similarly, the correlation model developed in the present study is described in the form:

$$
\text { and } \begin{aligned}
\Omega\left(\Delta^{+}, \tau^{+}\right) & =\Omega\left(\Delta_{x}^{+}, \tau^{+}\right) \cdot \Omega\left(\Delta_{y}^{+}\right) \cdot \Omega\left(\Delta_{z}^{+}\right), \\
\Omega\left(\Delta_{x}^{+}, \tau^{+}\right) & =\exp \left(-\sigma_{x 1} \pi\left|\Delta_{x}^{+}-\bar{u}^{+} \tau^{+}\right|^{b}-\sigma_{x 2} \pi\left|\Delta_{x}^{+}+\bar{u}^{+} \tau^{+}\right|^{b}\right), \\
\Omega\left(\Delta_{y}^{+}\right) & =\exp \left(-\sigma_{y} \pi \Delta_{y}^{+2}\right), \\
\Omega\left(\Delta_{z}^{+}\right) & =\exp \left(-\sigma_{z} \pi \Delta_{z}^{+b}\right) ;
\end{aligned}
$$

where $\bar{u}$ is the local mean streamwise velocity; the superscript + denotes a normalisation using the laminar flame thickness $\delta_{L}$ and laminar flame time $\tau_{L}$ as suggested in earlier studies (Swaminathan et al., 2011,a; Liu et al., 2012,a). In this correlation model (12), the correlation functions of $\Omega\left(\Delta_{y}^{+}\right)$and $\Omega\left(\Delta_{z}^{+}\right)$are standard Gaussian forms, whereas $\Omega\left(\boldsymbol{\Delta}^{+}, \tau^{+}\right)$takes a variant form due to the convection effect in the presence of the mean streamwise velocity. The first term in the exponent of Eq. (12b) is to ensure a symmetry to $\Delta_{x}^{+}=\bar{u}^{+} \tau^{+}$while the second term is to apply a decay of amplitude along $\Delta_{x}^{+}=\bar{u}^{+} \tau^{+}$, as will be shown in Section 4.4.

The coefficients $\sigma_{x 1}, \sigma_{x 2}, \sigma_{y}, \sigma_{z}$ in Eq. (12) denote the decay rate of $\Omega$ from unity to zero and are determined by fitting the models to the correlation function obtained from the DNS data. As listed in Table 2, two cases of the decay rates $\sigma_{x 1}, \sigma_{x 2}, \sigma_{y}, \sigma_{z}$ 
Table 2: Coefficients of decay rates $\sigma_{x 1}, \sigma_{x 2}, \sigma_{y}, \sigma_{z}$ and $b$ for the correlation model (12).

\begin{tabular}{cccccc}
\hline Case & $b$ & $\sigma_{x 1}$ & $\sigma_{x 2}$ & $\sigma_{y}$ & $\sigma_{z}$ \\
\hline 1 & 2.0 & 0.5 & 0.02 & 1.2 & 0.5 \\
2 & 1.6 & 0.4 & 0.025 & 1.2 & 0.4 \\
\hline
\end{tabular}

and $b$ have been considered and the purpose of using two sets of coefficients is to better match the DNS data in different regions of $\Delta_{x}^{+}$and $\Delta_{z}^{+}$. As can be seen in Table 2 , the values of $\sigma_{x 1}, \sigma_{x 2}, \sigma_{z}$ are slightly different between the two cases, whereas the coefficient $\sigma_{y}$ takes the same value for both cases. These coefficients may vary slightly with flame configurations and conditions, but the correlation model will retain the Gaussian function form regardless of the flame types. Moreover, it can be anticipated that flame annihilation will result in larger decay rates of this correlation through the enhancement of kinematic restoration effects and rapid destructions of flame surface area, and hence higher frequencies and lower amplitudes of sound sources. An important parameter that may affect the correlation function $\Omega$ is the Lewis number which was found to have a strong influence on the fluctuations in flame surface area and heat release rate and hence the resulting sound from flame annihilation, with larger Lewis numbers yielding more sound production (Talei et al., 2012, 2013). It can therefore be expected that large Lewis numbers will result in slow decay rates of the two-point space-time correlation and high sound levels.

In an earlier study (Liu et al., 2012), the two-point correlation of the rate of change of reaction rate fluctuation, $\ddot{w}^{\prime}$, was investigated using the same DNS data (Dunstan et al., 2011). It was found that the two-point correlation function $\Omega_{1}(\boldsymbol{\Delta})$, defined as

$$
\overline{\ddot{w}^{\prime}(\boldsymbol{y}, t) \ddot{w}^{\prime}(\boldsymbol{y}+\boldsymbol{\Delta})}=\Omega_{1}(\boldsymbol{\Delta}) \overline{\ddot{w}^{\prime 2}(\boldsymbol{y}, t)},
$$

can be well modelled by making use of Hermite-Gaussian functions of zero and second orders in the $\Delta_{x}$ direction, i.e.

$$
\Omega_{1}\left(\Delta_{x}^{+}\right)=\left(1-\epsilon_{x} \pi \Delta_{x}^{+2}\right) \exp \left(-\sigma_{x} \pi \Delta_{x}^{+2}\right),
$$

where the constants $\epsilon_{x}$ and $\sigma_{x}$ denote the decay rate of $\Omega_{1}\left(\Delta_{x}\right)$. The definitions of $\Omega$ and $\Omega_{1}$ in Eqs. (7b) and (13) indicate that the two correlation functions should be related through a double derivative with respect to the retarded time $\tau$,

$$
\frac{\partial^{2} \Omega}{\partial \tau^{2}}(\boldsymbol{\Delta}, 0)=\Omega_{1}(\boldsymbol{\Delta})
$$

The above relationship can be verified by applying it to the models of the correlation functions, $\Omega$ and $\Omega_{1}$, as in Eqs. (12b) and (14). Substituting Eq. (12b) for Case 1 into Eq. (15) and recognising that $\Omega(0,0)=1$, one obtains the validated form of Hermite-Gaussian functions in Eq. (14) (Liu et al., 2012). Hence the proposed correlation model of $\Omega$ satisfies the relationship (15), which implies that the model form of the coupled space-time correlation function $\Omega\left(\Delta_{x}^{+}, \tau^{+}\right)$is verified mathematically. Case 2 is a variant of Case 1 and will produce very similar results as will be shown in subsequent sections in which the correlation model of both cases will be compared to the results from the V-flame DNS data. 


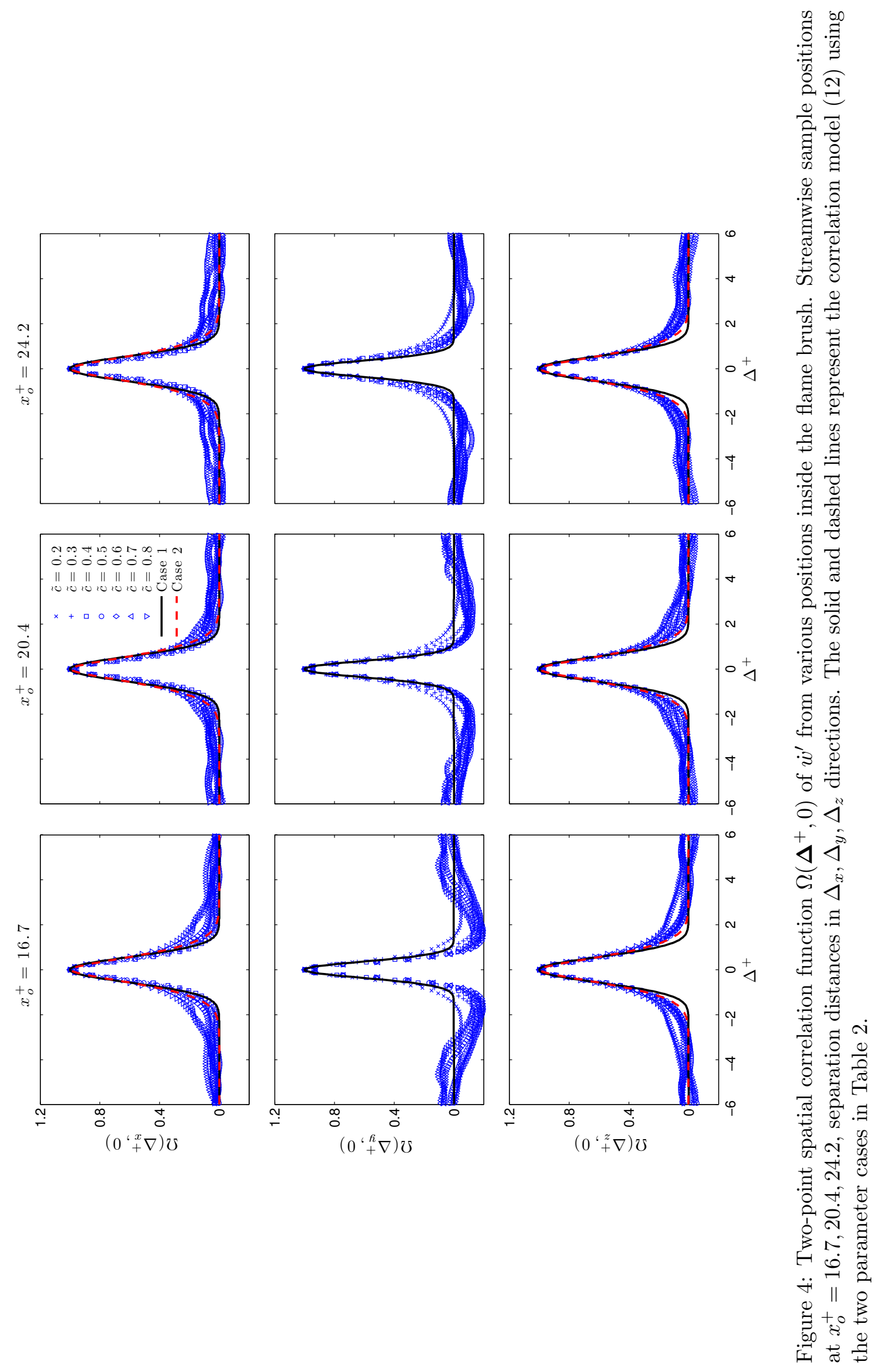




\subsection{Spatial correlation}

Consider first the two-point spatial correlation function $\Omega\left(\boldsymbol{\Delta}^{+}, 0\right)$. As shown in Figure 4, the separation distances $\Delta_{x}, \Delta_{y}$ and $\Delta_{z}$ are taken in the streamwise, transverse and spanwise directions, respectively. For each direction, three streamwise positions at $x_{o}^{+}=$ $16.7,20.4,24.2$ and seven transverse positions at $\tilde{c}=0.2,0.3, \ldots, 0.8$ are taken, yielding a total of 21 groups of data. It can be observed that the correlation function $\Omega$ is symmetric about $\Delta^{+}=0$ for all positions and directions. The value of $\Omega$ drops quickly from 1 to 0.05 within one to two laminar flame thickness $\delta_{L}$. This abrupt decay of the spatial correlation of $\dot{w}^{\prime}$ can be explained by the dynamics of small scale turbulence that controls predominantly the dynamics and fluctuations of the chemical reactions in thin regions (Swaminathan et al., 2011a). The rate of decay is observed to be different in the $\Delta_{x}, \Delta_{y}, \Delta_{z}$ directions, which is consistent with the findings in Liu et al. (2012). As can be seen in Figure 4, the decay rates in the $\Delta_{x}$ and $\Delta_{z}$ directions are very similar which is confirmed by the correlation model form (12) and the values of $\sigma_{x 1}, \sigma_{x 2}, \sigma_{z}$ in Table 2. The decay rate in the $\Delta_{y}$ direction, however, is somewhat faster than the other two directions, as a result of the larger transverse gradients of reaction rate fluctuations $\dot{w}^{\prime}$ within the relatively shorter span of the flame brush in this direction (Liu et al., 2012).

As shown in Figure 4, the Gaussian function given in the correlation model (12) represents reasonably well the correlation function $\Omega\left(\boldsymbol{\Delta}^{+}\right)$evaluated from the DNS results. In the $\Delta_{x}$ and $\Delta_{z}$ directions, the curve fits are shown as solid and dashed lines for the two cases of coefficients, respectively. Case 2 appears to represent the data better in terms of the broadening of the modelled curves particularly for $\Omega\left(\Delta_{z}^{+}\right)$at large values $\left|\Delta_{z}^{+}\right|>1$, whereas Case 1 fits the data slightly better when $\left|\Delta_{z}^{+}\right|$is close to zero as manifested in the diminished gradient of the curve. A very small variation of $\Omega\left(\boldsymbol{\Delta}^{+}\right)$ with $x_{o}^{+}$and $\tilde{c}$ confirms that this correlation function is independent on spatial positions (Swaminathan et al., 2011a; Liu et al., 2012). However, the Gaussian function does not fit $\Omega\left(\Delta^{+}\right)$very well in the transverse $\Delta_{y}^{+}$direction; the correlation function $\Omega\left(\Delta_{y}^{+}\right)$from the DNS data takes negative values as small as -0.2 around $\left|\Delta_{y}^{+}\right|=2$. This negative zone appears to diminish gradually for further downstream locations $\left(x_{o}^{+}=24.2\right)$ which correspond to a wider spread of the flame brush and a better mixed region of the flow. Another possible reason for the negative values is that the statistical samples available for averaging are insufficient because these oscillations are found to increase by a halved sample size. In addition, the DNS results of $\Omega\left(\Delta_{x}^{+}\right)$show broad 'tails' with relatively slow decay around $\left|\Delta_{x}^{+}\right|=2$ for the sample position at $x_{o}^{+}=16.7$. These tails disappear gradually as the sample point moves into the flame brush or when $x_{o}^{+}$ moves downstream, yielding a better collapse of the spatial correlation which can be ascribed to the better mixing inside the flame brush and in the downstream region. Nevertheless, the theoretical model of the spatial correlation function $\Omega\left(\boldsymbol{\Delta}^{+}\right)$can be regarded as acceptable in reproducing the DNS data.

\subsection{Temporal correlation}

Consider next the two-point temporal correlation function $\Omega\left(0, \tau^{+}\right)$. Similar to the spatial correlation $\Omega\left(\boldsymbol{\Delta}^{+}, 0\right)$ in Figure 4 , the results of $\Omega\left(0, \tau^{+}\right)$are shown in Figure 5 for the three $x_{o}^{+}$positions and seven $\tilde{c}$ values or cross-stream positions. Similar features 

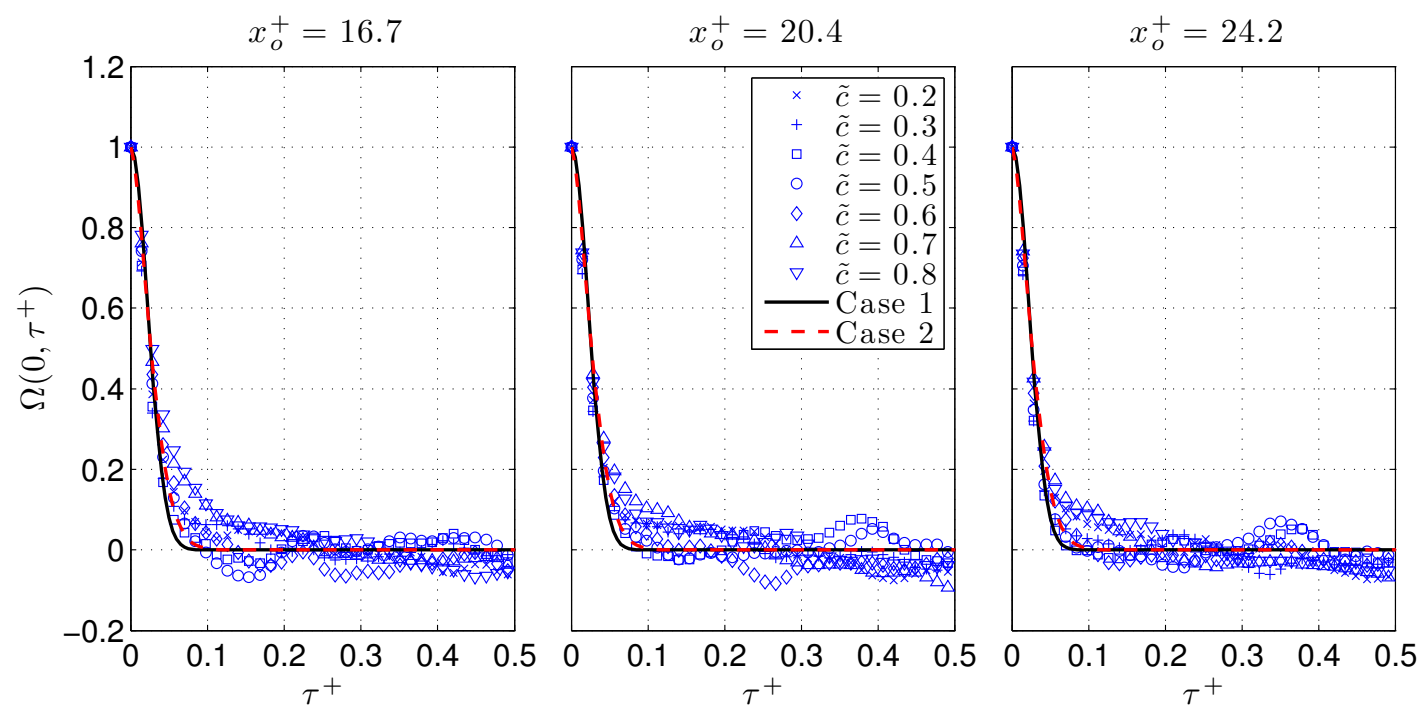

Figure 5: Two-point temporal correlation function $\Omega\left(0, \tau^{+}\right)$of reaction rate fluctuations from various positions inside the flame brush. The solid and dashed lines represent the correlation model (12) using the two sets of coefficients in Table 2.

can be observed for the temporal correlation function $\Omega\left(\tau^{+}\right)$. The $\Omega$ value decays sharply within a very short time. The average value over the transverse positions reaches $\Omega=0.05$ at around $\tau^{+}=0.1$. The rapid decay of $\Omega\left(\tau^{+}\right)$can be attributed to the abrupt variation of the reaction rate fluctuations $\dot{w}^{\prime}$ with time when the flame front sweeps over that position, as have been shown in Figure 3. Negative values of $\Omega$ also exist due to fewer statistical samples available for time averaging, i.e. $N_{\text {ave }}<N_{t}$. The Gaussian function in Eq. (12) for the correlation now reduces to

$$
\Omega\left(\tau^{+}\right)=\exp \left[-\left(\sigma_{x 1}+\sigma_{x 2}\right) \pi\left|\bar{u}^{+} \tau^{+}\right|^{b}\right],
$$

and it approximates the DNS results well. Out of the two sets of coefficients in Table 2, Case 2 approximates the data slightly better in the range $0.03<\tau^{+}<0.1$, and the difference between the curve fits of the two cases is nearly negligible in other $\tau^{+}$regions. In addition, the spatial independence is observed because $\Omega\left(\tau^{+}\right)$only varies slightly with positions. Similar to the spatial correlation, a slightly better collapse of the DNS data onto the correlation model can be observed inside the flame brush and at downstream sample positions due to the faster flow mixing in these regions.

\subsection{Spatio-temporal correlation}

Figures 6(a)-6(c) illustrate the contours of the spatio-temporal correlation function $\Omega\left(\boldsymbol{\Delta}^{+}, \tau^{+}\right)$for the three directions. Again, both the DNS and modelled data of $\Omega\left(\boldsymbol{\Delta}^{+}, \tau^{+}\right)$are shown for comparison. As can be seen from the top two figures 6(a) and $6(\mathrm{~b})$, in the $\left(\Delta_{y}^{+}, \tau^{+}\right)$and $\left(\Delta_{z}^{+}, \tau^{+}\right)$planes this correlation function can be simply modelled by

$$
\Omega\left(\Delta^{+}, \tau^{+}\right)=\Omega\left(\Delta^{+}, 0\right) \cdot \Omega\left(0, \tau^{+}\right)
$$

because of the separable functions $\Omega\left(\Delta_{y}^{+}\right)$and $\Omega\left(\Delta_{z}^{+}\right)$in the model of the correlation function given in Eq. (12). The distribution of contours in the two planes is symmetric 

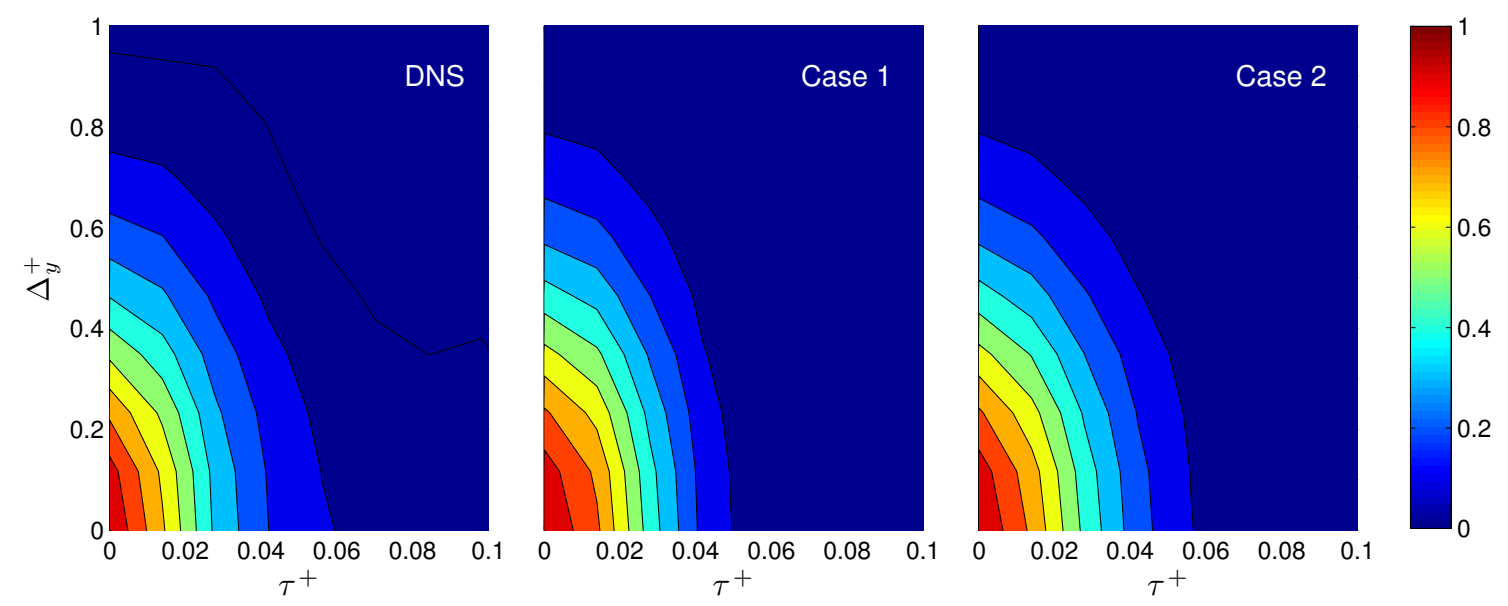

(a) $\left(\Delta_{y}^{+}, \tau^{+}\right)$plane, $\Delta_{x}^{+}=0, \Delta_{z}^{+}=0$
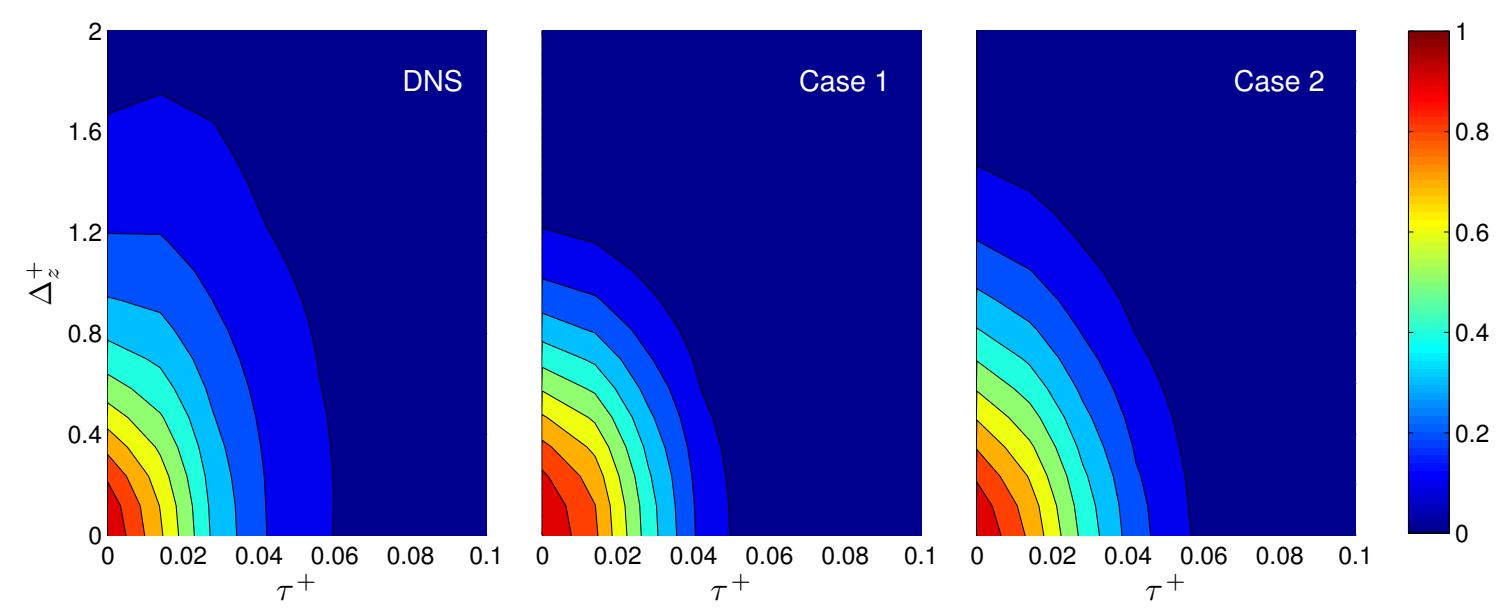

(b) $\left(\Delta_{z}^{+}, \tau^{+}\right)$plane, $\Delta_{x}^{+}=0, \Delta_{y}^{+}=0$
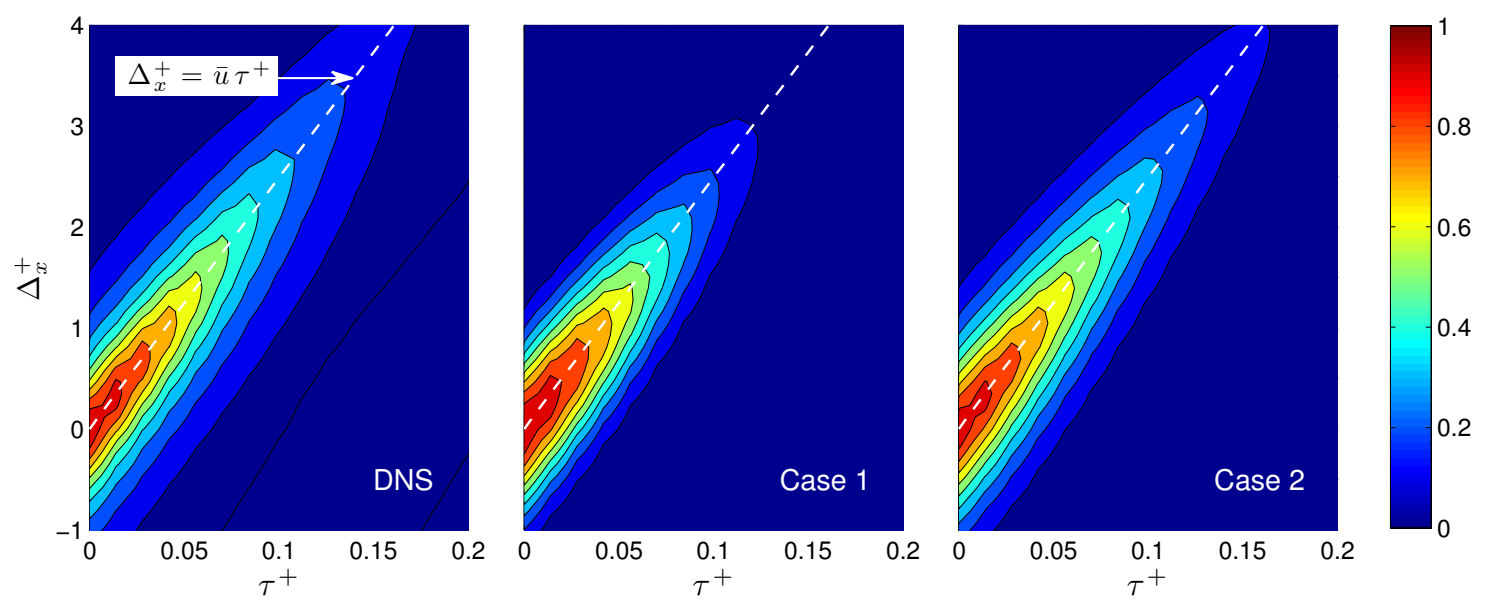

(c) $\left(\Delta_{x}^{+}, \tau^{+}\right)$plane, $\Delta_{y}^{+}=0, \Delta_{z}^{+}=0$

Figure 6: Contours of the correlation functions of reaction rate fluctuations in the $\left(\boldsymbol{\Delta}^{+}, \tau^{+}\right)$planes and comparison between the DNS data and the model in (12). Sample point at $x_{o}^{+}=16.7, \tilde{c}=0.5$. 
to $\Delta^{+}=0$ and $\tau^{+}=0$, and thus only the positive regions of $\Delta^{+}$and $\tau^{+}$are shown in Figures 6(a) and 6(b). Compared to the spatial correlation $\Omega\left(\Delta^{+}\right)$, the temporal correlation $\Omega\left(\tau^{+}\right)$attenuates the amplitude of $\Omega\left(\Delta^{+}, \tau^{+}\right)$linearly but does not change the location of the peak at $\Delta^{+}=0$.

For the streamwise $x$ direction, the spatial and temporal correlations are coupled with each other and so the correlation function $\Omega\left(\Delta_{x}^{+}, \tau^{+}\right)$is inseparable, i.e.

$$
\Omega\left(\Delta_{x}^{+}, \tau^{+}\right) \neq \Omega\left(\Delta_{x}^{+}, 0\right) \cdot \Omega\left(0, \tau^{+}\right)
$$

in the presence of the mean streamwise velocity $\bar{u}^{+}$. An obvious convection effect with the mean flow velocity $\bar{u}^{+}$can be observed from the bottom figure $6(\mathrm{c})$ featured by the symmetric distribution of the $\Omega\left(\Delta_{x}^{+}, \tau^{+}\right)$contours with respect to $\Delta_{x}^{+}=\bar{u}^{+} \tau^{+}$, where $\bar{u}^{+}=24.5$ at the sample point $x_{o}^{+}=16.7, \tilde{c}=0.5$. This symmetry to $\Delta_{x}^{+}=\bar{u}^{+} \tau^{+}$is achieved through the first term in the exponent of Eq. (12b), while the amplitude decay along $\Delta_{x}^{+}=\bar{u}^{+} \tau^{+}$is produced by the second term in the exponent. Again the correlation function $\Omega$ drops rapidly as either $\left|\Delta_{x}^{+}-\bar{u}^{+} \tau^{+}\right|$or $\left|\Delta_{x}^{+}+\bar{u}^{+} \tau^{+}\right|$increases. In contrast to the correlation functions $\Omega\left(\Delta_{y}^{+}, \tau^{+}\right)$and $\Omega\left(\Delta_{z}^{+}, \tau^{+}\right)$shown in Figures 6(a) and 6(b), apart from the amplitude attenuation the temporal correlation $\Omega\left(\tau^{+}\right)$also alters the peak of $\Omega\left(\Delta_{x}^{+}, \tau^{+}\right)$to the location $\Delta_{x}^{+}=\bar{u}^{+} \tau^{+}$. Moreover, the overall agreement of the model correlation function $\Omega\left(\boldsymbol{\Delta}^{+}, \tau^{+}\right)$with the DNS contours is noteworthy, particularly for the Case 2 coefficients, given the strong convection effect in the streamwise direction.

Figure 7 shows the spatio-temporal correlation function $\Omega\left(\Delta_{x}^{+}, \tau^{+}\right)$at the location $x_{o}^{+}=16.7, \tilde{c}=0.5$ processed from the same DNS data as in Figure 6(c). It is obviously shown in Figure 7 that the peak of the correlation function, $\Omega_{p}\left(\Delta_{x}^{+}, \tau^{+}\right)$, shifts to larger separation distances $\Delta_{x}^{+}$with increasing values of $\tau^{+}$. The location and time delay

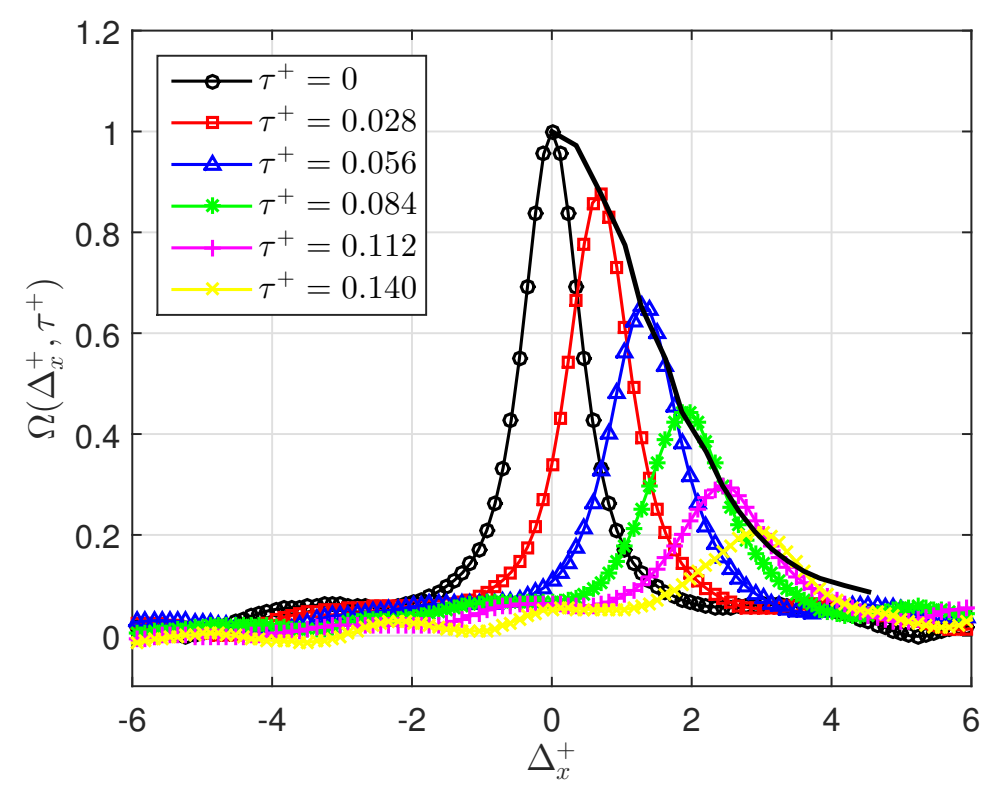

Figure 7: Two-point spatial correlation function $\Omega\left(\Delta_{x}^{+}\right)$at various values of $\tau^{+}$at the sample position $x_{o}^{+}=16.7, \tilde{c}=0.5$. The solid line shows the envelope of peak correlation values $\Omega_{p}\left(\Delta_{x}^{+}, \tau^{+}\right)$. 


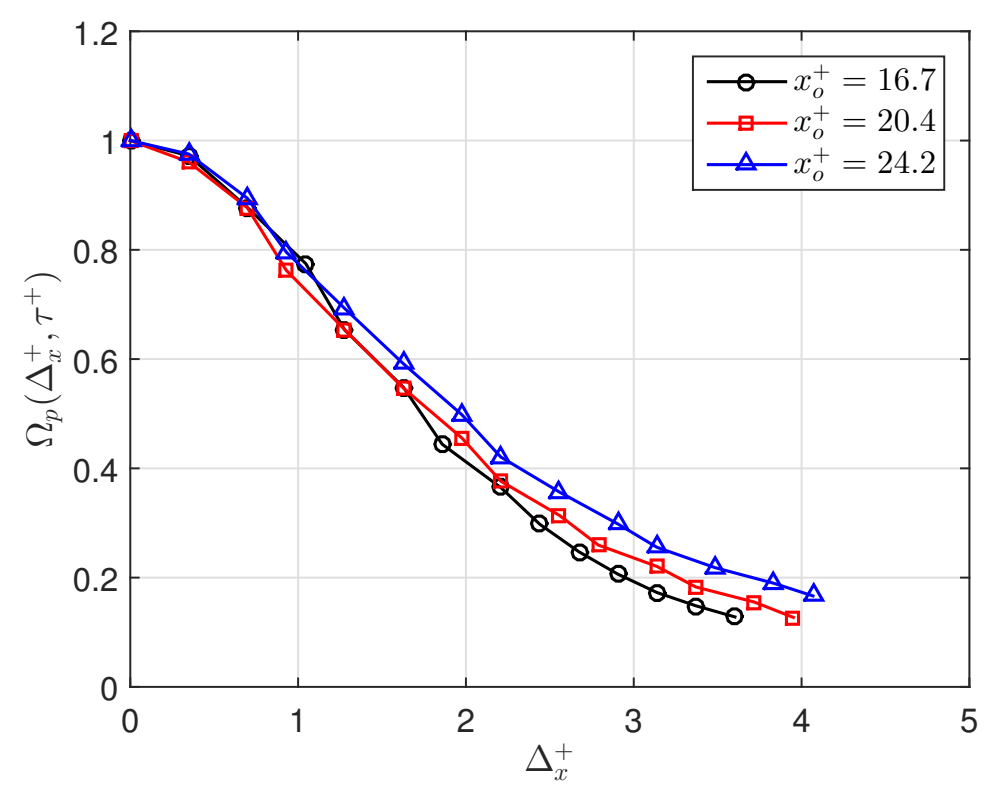

Figure 8: Peak correlation envelopes of reaction rate fluctuations at different streamwise sample positions, $\tilde{c}=0.5$.

of the peak correlation are determined by $\Delta_{x}^{+}=\bar{u}^{+} \tau^{+}$, which is in agreement with the convection characteristics observed in Figure 6(c) as well as the spatio-temporal correlations of jet noise sources (e.g. Karabasov et al., 2010, 2011; Depuru Mohan et al., 2015), local burning area fluctuations (Hemchandra and Lieuwen, 2010), and local chemiluminescence fluctuations (Rajaram and Lieuwen, 2009). The envelope of the peak correlation values indicates the decay of $\Omega_{p}\left(\Delta_{x}^{+}, \tau^{+}\right)$with the separation distance $\Delta_{x}^{+}$and/or time delay $\tau^{+}$. This behaviour of the spatio-temporal correlation is also observed at other sample points. Figure 8 shows the envelopes of the peak correlation $\Omega_{p}\left(\Delta_{x}^{+}, \tau^{+}\right)$obtained at the three streetwise sample positions $x_{o}^{+}=16.7,20.4,24.2$. The decay rate of the peak correlation generally decreases as the sample position moves downstream, and this trend agrees qualitatively with the theoretical/numerical results for the spatio-temporal correlation of local burning area fluctuations (Hemchandra and Lieuwen, 2010) and the experimental data of the spatio-temporal correlation of local heat release rate fluctuations using $\mathrm{CH}^{*}$ chemiluminescence (Rajaram and Lieuwen, 2009).

\subsection{Spectrum of correlation function}

The temporal correlation of heat release rate fluctuations provides information to obtain the frequency spectrum of the correlation model (12) through a Fourier transform of the time-domain results of $\Omega\left(\Delta_{x}^{+}, \tau^{+}\right)$,

$$
\hat{\Omega}^{+}\left(\Delta_{x}^{+}, \omega^{+}\right)=\int_{-\infty}^{\infty} \Omega\left(\Delta_{x}^{+}, \tau^{+}\right) \mathrm{e}^{-\mathrm{i} \omega^{+} \tau^{+}} \mathrm{d} \tau^{+} .
$$

The correlation functions $\Omega\left(\Delta_{y}^{+}\right)$and $\Omega\left(\Delta_{z}^{+}\right)$are ignored in the Fourier transform because they are independent of $\tau^{+}$and hence do not contribute to the frequency 


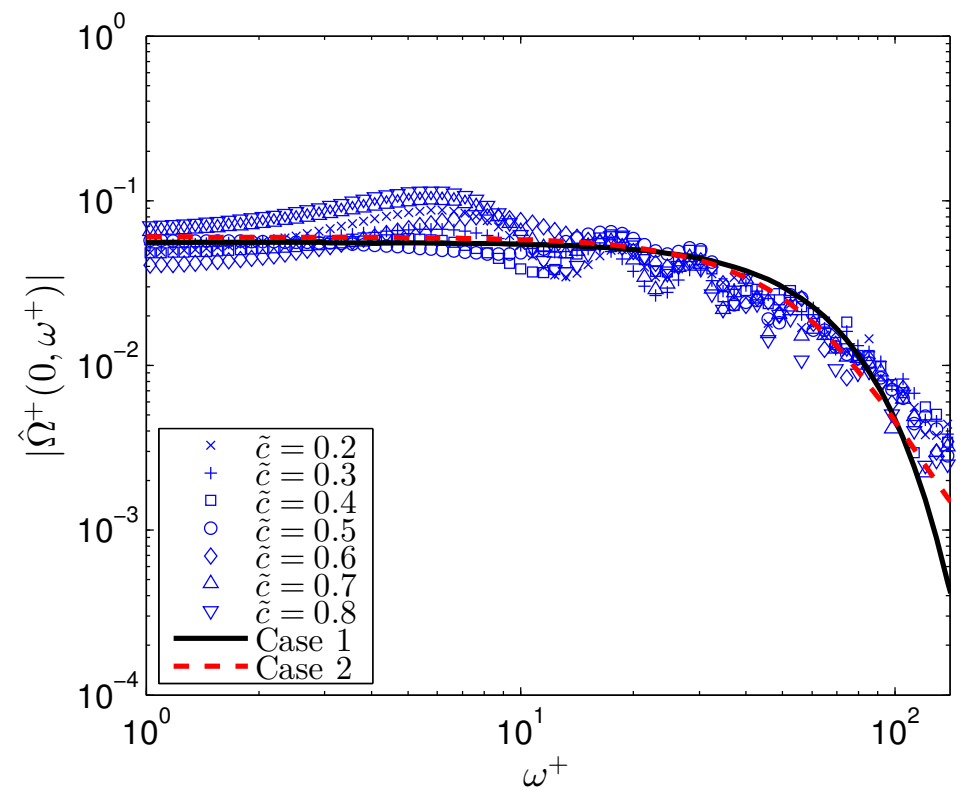

Figure 9: Spectrum of the temporal correlation function $\Omega\left(0, \tau^{+}\right)$for sample positions at $x_{o}^{+}=16.7, \tilde{c}=0.2,0.3, \ldots, 0.8$. The solid and dashed lines represent the spectra of the correlation model (12) using the two cases in Table 2.

dependence of the correlation function $\Omega\left(\boldsymbol{\Delta}^{+}, \tau^{+}\right)$. Figure 9 shows the spectrum of the correlation function, $\hat{\Omega}^{+}\left(0, \omega^{+}\right)$, for one streamwise position at $x^{+}=16.7$ and the seven $\tilde{c}$ values along with the comparison between the data of the DNS and the model. It is shown that the correlation spectrum $\hat{\Omega}$ is flat at low frequencies and then it begins to drop off around non-dimensional frequency $\omega^{+}=40$. Similar results of a $\omega^{0}$ dependence in the low-frequency spectrum of heat release fluctuations have been observed in the measurements by Rajaram and Lieuwen (2009). The slight variation of $\hat{\Omega}$ among the different transverse positions indicates that the spatial independence of $\Omega\left(\boldsymbol{\Delta}^{+}, \tau^{+}\right)$observed in Sections 4.2 and 4.3 is retained in the frequency domain.

The spectral characteristics of $\hat{\Omega}$ from the DNS results are well captured by the Gaussian functions in the correlation model (12). The two sets of coefficients $\sigma_{x 1}, \sigma_{x 2}, \sigma_{y}, \sigma_{z}$ and $b$ give very close results of $\hat{\Omega}^{+}\left(0, \omega^{+}\right)$and the difference is nearly negligible in terms of SPL $(\mathrm{dB})$ at frequencies up to $\omega^{+}=100$. Above this frequency the coefficients of Case 2 produces slightly closer agreement with the DNS data. In particular, the flat spectral shape at low frequencies will enable the modelling of the spectral peak of combustion noise when multiplying the term $\omega^{2}$ as in the PSD expression (9). The $\omega^{0}$ dependence in the low-frequency spectrum of $\hat{\Omega}$ multiplied by the term $\omega^{2}$ will result in a constant low-frequency slope of $\beta=2$ for the predicted noise spectrum, which is consistent with the slope found in experimental data (Rajaram, 2007; Rajaram and Lieuwen, 2009).

The contours of the correlation spectrum $\hat{\Omega}^{+}\left(\Delta_{x}^{+}, \omega\right)$ are illustrated in Figure 10 and compared between the DNS results and correlation model (12). The coefficients of Case 2 fit the DNS contours better, as expected, due to the better agreement in the spatio-temporal contours $\Omega\left(\Delta_{x}^{+}, \tau^{+}\right)$(see Figure 6(c)). Note that the coupled space-time 

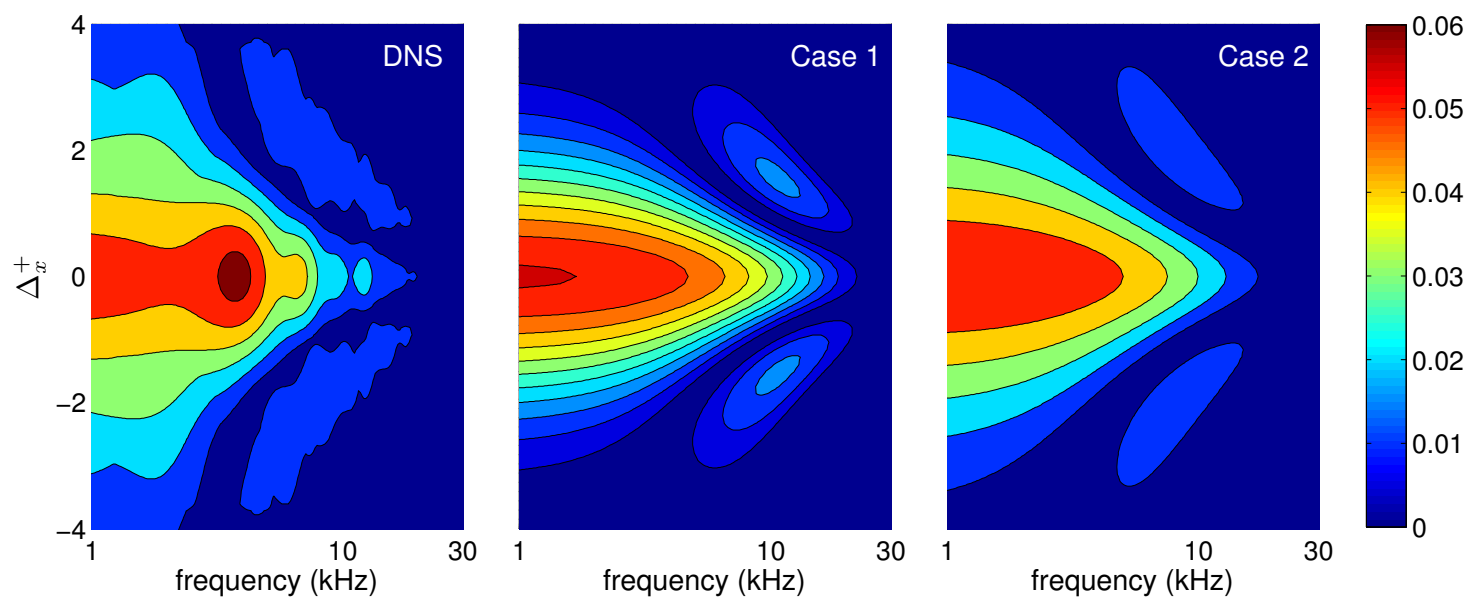

Figure 10: Contours of the spectrum of the correlation function, $\hat{\Omega}^{+}\left(\Delta_{x}^{+}, \omega\right)$, and comparison between the DNS data and the model in (12). Sample point at $x^{+}=16.7$, $\tilde{c}=0.5$.

correlation function $\Omega\left(\Delta_{x}^{+}, \tau^{+}\right)$is symmetric to $\Delta_{x}^{+}=\bar{u}^{+} \tau^{+}$as shown in Figure 6(c), whereas after the Fourier transform its spectrum $\hat{\Omega}^{+}\left(\Delta_{x}^{+}, \omega\right)$ becomes symmetric to $\Delta_{x}^{+}=0$ in the frequency domain. This is because $\Omega\left(\Delta_{x}^{+}, \tau^{+}\right)$is also symmetric to the norm of $\Delta_{x}^{+}=\bar{u}^{+} \tau^{+}$, and hence $\Omega\left(\Delta_{x}^{+}, \tau^{+}\right)=\Omega\left(-\Delta_{x}^{+},-\tau^{+}\right)$. At a specific frequency, the correlation spectrum $\hat{\Omega}^{+}\left(\Delta_{x}^{+}, \omega\right)$ drops rapidly as $\left|\Delta_{x}\right|$ increases; the peak of $\hat{\Omega}^{+}$ always occurs at $\Delta_{x}^{+}=0$ and its variation with frequency is consistent with the frequency dependence observed in Figure 9. Moreover, as frequency increases the contour region of $\hat{\Omega}^{+}\left(\Delta_{x}^{+}, \omega\right)$ narrows gradually in the $\Delta_{x}^{+}$direction, which indicates that $\hat{\Omega}^{+}$drops more quickly with $\Delta_{x}^{+}$at higher frequencies.

\subsection{Integral length scales and correlation volume}

The integral length scale for $\dot{w}^{\prime}$ is defined as

$$
\ell^{+}\left(\tau^{+}\right)=\frac{1}{2} \int_{-\infty}^{\infty} \Omega\left(\Delta^{+}, \tau^{+}\right) \mathrm{d} \Delta^{+} .
$$

In contrast to the definition of $\ell^{+}$for the rate of change of reaction rate fluctuations, $\ddot{w}$, as reported in Swaminathan et al. (2011a) and Liu et al. (2012), the integral length scale for $\dot{w}^{\prime}$ has a dependence on $\tau^{+}$. In the transverse and spanwise directions, Eq. (20) can be simplified as

$$
\ell_{y, z}^{+}\left(\tau^{+}\right)=\Omega\left(\tau^{+}\right) \int_{0}^{\infty} \Omega\left(\Delta_{y, z}^{+}\right) \mathrm{d} \Delta_{y, z}^{+}
$$

because $\Omega\left(\Delta_{y}^{+}\right)$and $\Omega\left(\Delta_{z}^{+}\right)$are symmetric to $\Delta^{+}=0$ and can be separated from $\Omega\left(\boldsymbol{\Delta}^{+}, \tau^{+}\right)$as shown in Eq. (12a). Using the Gaussian function model for $\Omega$ in Eq. (12) and the Case 1 coefficient $(b=2)$, one can calculate analytically the integral length scales in the $y, z$-directions, i.e.

$$
\begin{aligned}
\ell_{y}^{+}\left(\tau^{+}\right) & =\frac{1}{2} \sigma_{y}^{-1 / 2} \Omega\left(\tau^{+}\right) \\
\text {and } \quad \ell_{z}^{+}\left(\tau^{+}\right) & =\frac{1}{2} \sigma_{z}^{-1 / 2} \Omega\left(\tau^{+}\right)
\end{aligned}
$$




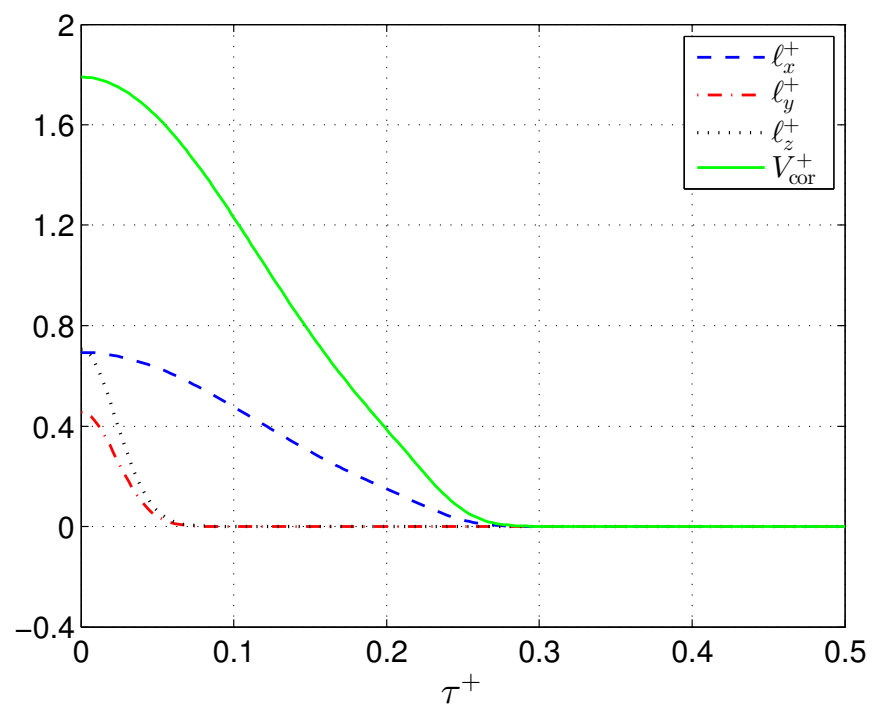

Figure 11: Time-dependent integral length scales $\ell_{x}^{+}, \ell_{y}^{+}, \ell_{z}^{+}$and correlation volume $V_{\text {cor }}^{+}$ for Case 1 of the correlation model (12).

with $\Omega\left(\tau^{+}\right)$given in Eq. (16), while the evaluation of the integral length scale in the streamwise direction $\ell_{x}^{+}$needs a numerical integration of Eq. (20). The Case 2 coefficient, $b=1.6$, does not lead to an analytical solution of the integral in Eq. (21). The time-dependent correlation volume can be obtained by integrating $\Omega$ over $v_{\text {cor }}$, i.e.

$$
V_{\text {cor }}^{+}\left(\tau^{+}\right)=\int_{v_{\text {cor }}} \Omega\left(\boldsymbol{\Delta}^{+}, \tau^{+}\right) \mathrm{d}^{3} \boldsymbol{\Delta}^{+}=8 \ell_{x}^{+}\left(\tau^{+}\right) \ell_{y}^{+}(0) \ell_{z}^{+}(0) .
$$

Substituting Eq. (22) into Eq. (23), the integral correlation volume can be expressed as

$$
V_{\text {cor }}^{+}\left(\tau^{+}\right)=\sigma_{y}^{-1 / 2} \sigma_{z}^{-1 / 2} \int_{-\infty}^{\infty} \Omega\left(\Delta_{x}^{+}, \tau^{+}\right) \mathrm{d} \Delta_{x}^{+} .
$$

Figure 11 shows the integral length scales $\ell_{x}^{+}, \ell_{y}^{+}, \ell_{z}^{+}$and correlation volume $V_{\text {cor }}^{+}$ as a function of $\tau^{+}$using the Case 1 coefficients in the correlation model (12). The integral length scales $\ell_{y}^{+}, \ell_{z}^{+}$vary similarly as $\Omega\left(\tau^{+}\right)$(see Figure 5); they drop from the peaks at $\tau^{+}=0$ and reach values close to zero around $\tau^{+}=0.08$. The integral length scale $\ell_{x}^{+}$, however, displays a much slower decay with time and becomes close to zero around $\tau^{+}=0.28$; the trend of the correlation volume $V_{\text {cor }}^{+}\left(\tau^{+}\right)$is identical to $\ell_{x}^{+}\left(\tau^{+}\right)$ according to Eq. (23). This is because for a given $\Delta_{x}^{+}$the location of the peak correlation $\Omega_{p}\left(\Delta_{x}^{+}, \tau^{+}\right)$shifts to $\tau^{+}=\Delta_{x}^{+} / \bar{u}^{+}$, and hence $\Omega\left(\Delta_{x}^{+}, \tau^{+}\right)$remains non-negligible at large $\tau^{+}$. Moreover, the peak values of $\ell_{x}^{+}, \ell_{y}^{+}, \ell_{z}^{+}$and $V_{\text {cor }}^{+}$all occur at $\tau^{+}=0$ and they are dependent on the decay rates of the spatial correlation function $\Omega\left(\boldsymbol{\Delta}^{+}, 0\right)$ as shown in Figure 4 and Table 2 . The very close peak values $\ell_{x}^{+}(0)$ and $\ell_{z}^{+}(0)$ are due to the similar decay rates of $\Omega\left(\boldsymbol{\Delta}^{+}, 0\right)$ in the $\Delta_{x}$ and $\Delta_{z}$ directions.

Similarly, the frequency spectra of the integral length scales and correlation volume can be obtained by applying an FT to $\ell^{+}\left(\tau^{+}\right)$and $V_{\text {cor }}^{+}\left(\tau^{+}\right)$in time domain. It is obvious from Eq. (22) that the spectra of the length scales $\ell_{y}^{+}, \ell_{z}^{+}$are proportional to the spectrum of the correlation function, $\hat{\Omega}^{+}\left(\omega^{+}\right)$, which can be found in Figure 9 . 


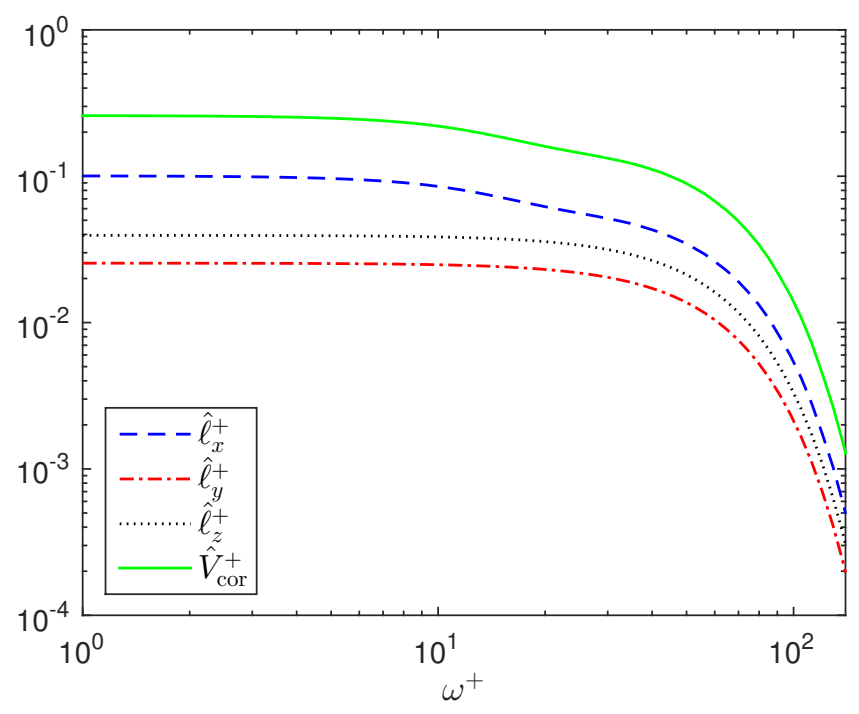

Figure 12: Spectra of the integral length scales $\ell_{x}^{+}, \ell_{y}^{+}, \ell_{z}^{+}$and correlation volume $V_{\text {cor }}^{+}$ for Case 1 of the correlation model (12).

The spectra of the length scale in the $x$-direction and the correlation volume can be calculated via

$$
\hat{\ell}_{x}^{+}\left(\omega^{+}\right)=\frac{1}{2} \int_{-\infty}^{\infty} \hat{\Omega}^{+}\left(\Delta_{x}^{+}, \omega^{+}\right) \mathrm{d} \Delta_{x}^{+}
$$

and

$$
\hat{V}_{\text {cor }}^{+}\left(\omega^{+}\right)=\sigma_{y}^{-1 / 2} \sigma_{z}^{-1 / 2} \int_{-\infty}^{\infty} \hat{\Omega}^{+}\left(\Delta_{x}^{+}, \omega^{+}\right) \mathrm{d} \Delta_{x}^{+},
$$

where the data of $\hat{\Omega}^{+}\left(\Delta_{x}^{+}, \omega^{+}\right)$are presented in Figure 10. The results of the frequency-dependent integral length scales $\ell_{x}^{+}, \ell_{y}^{+}, \ell_{z}^{+}$and correlation volume $V_{\text {cor }}^{+}$are shown in Figure 12 using the Case 1 coefficients in the correlation model (12). The spectra of the integral length scales $\ell_{y}^{+}, \ell_{z}^{+}$retain the flat low-frequency dependence of $\hat{\Omega}^{+}\left(\omega^{+}\right)$in Figure 9. The trends of $\hat{\ell}_{x}^{+}\left(\omega^{+}\right)$and $\hat{V}_{\text {cor }}^{+}\left(\omega^{+}\right)$are very similar since they both include an integral of $\hat{\Omega}^{+}\left(\Delta_{x}^{+}, \omega^{+}\right)$with respect to $\Delta_{x}^{+}$. The decay of $\hat{\ell}_{x}^{+}$and $\hat{V}_{\text {cor }}^{+}$ as frequency increases is attributed to the narrowing contour region of $\hat{\Omega}^{+}\left(\Delta_{x}^{+}, \omega\right)$ with increasing frequency as can be seen in Figure 10.

\section{Conclusions}

The temporal correlation of the heat release rate fluctuation is crucial in predicting the spectral characteristics of combustion noise. This paper investigates the two-point space-time correlation of the heat release rate fluctuations inside the flame brush by analysing high-fidelity DNS data of turbulent premixed V-flames (Dunstan et al., 2011). The purposes are to understand the characteristics of this correlation and the associated correlation volume and its length scale, and to develop a theoretical model for the correlation function.

The two-point space-time correlation function $\Omega(\boldsymbol{\Delta}, \tau)$ are evaluated at various positions within the flame brush and the results have shown that this correlation drops 
rapidly from unity with both $\Delta$ and $\tau$ due to the abrupt fluctuation of $\dot{Q}$. This correlation function has also shown different decay rates in $\Delta_{x}, \Delta_{y}, \Delta_{z}$ directions and an independence on spatial positions inside the flame brush. Furthermore, the correlation function $\Omega(\boldsymbol{\Delta}, \tau)$ can be well approximated by Gaussian-type functions in both space and time except in the transverse direction. A strong convection effect is observed in the streamwise direction and this is also captured in the correlation model. In this case the correlation function $\Omega\left(\Delta_{x}^{+}, \tau^{+}\right)$is symmetric to $\Delta_{x}^{+}=\bar{u}^{+} \tau^{+}$with coupled spatial and temporal correlations. The integral length scales and correlation volume for the heat release rate fluctuation obtained using this model are time dependent with the peaks occurring at $\tau^{+}=0$. The integral length scales in the sreamwise direction, $\ell_{x}^{+}$, and the correlation volume $V_{\text {cor }}^{+}$decay much more slowly with time than the integral length scales $\ell_{y}^{+}, \ell_{z}^{+}$because of the convection effect. The peak values of $\ell_{x}^{+}, \ell_{y}^{+}, \ell_{z}^{+}$and $V_{\text {cor }}^{+}$are determined by the decay rates of the spatial correlation function $\Omega\left(\boldsymbol{\Delta}^{+}, 0\right)$.

The spectrum of the correlation function, $\hat{\Omega}^{+}\left(\omega^{+}\right)$, has shown a flat low-frequency dependence, which is consistent with the observed $\omega^{0}$ dependence of heat release fluctuations at low frequencies in previous experiments (Rajaram and Lieuwen, 2009). This flat spectral shape in the low-frequency range will also allow the appearance of a spectral peak and a low-frequency slope $\beta=2$ when multiplying $\hat{\Omega}$ by $\omega^{2}$ to model the spectral shape of combustion noise. The flat low-frequency dependence is retained in the spectra of the integral length scales $\ell_{y}^{+}$and $\ell_{z}^{+}$. The spectra of $\hat{\ell}_{x}^{+}$and $\hat{V}_{\text {cor }}^{+}$decay with frequency because $\hat{\Omega}^{+}\left(\Delta_{x}^{+}, \omega\right)$ contours gradually narrow as the frequency increases.

This work represents an explorative modelling of the two-point space-time correlation of heat release rate fluctuations in turbulent premixed flames, its associated correlation volume and length scale along with the frequency dependence, and it shows the potential of this correlation function for applying to predict the spectral shape of combustion noise. Further work on a longer DNS signal with more time steps is suggested to reduce the oscillations and negative values observed for $\Omega(\boldsymbol{\Delta}, \tau)$. In addition, an appropriate reference time is required to scale the peak frequency of combustion noise spectrum. These issues need to be addressed before this methodology can be applied to capture the full spectral characteristics of direct combustion noise (for example, spectral level and peak frequency).

\section{Acknowledgements}

The work was supported by the UK Technology Strategy Board under contract TP11/HVM/6/I/AB201K. Dr Dunstan's help while acquiring the V-flame DNS data is acknowledged. The author is grateful to Prof. Ann Dowling and Prof. N. Swaminathan at the University of Cambridge for lots of helpful discussions and suggestions. 


\section{References}

Alshaalan, T. M. and Rutland, C. J. (1998), Turbulence, scalar transport, and reaction rates in flame-wall interaction, in 'Twenty-Seventh Symposium (International) on Combustion', pp. 793-799.

Balachandran, R., Ayoola, B. O., Kaminski, C. F., Dowling, A. P. and Mastorakos, E. (2005), 'Experimental investigation of the nonlinear response of turbulent premixed flames to imposed inlet velocity oscillations', Combust. Flame 143, 37-55.

Bell, J. B., Day, M. S., Shepherd, I. G., Johnson, M. R., Cheng, R. K., Grcar, J. F., Beckner, V. E. and Lijewski, M. (2005), 'Numerical simulations of a laboratory-scale turbulent V-flame', Proc. Natl. Acad. Sci. 102(29), 10006-10011.

Bragg, S. L. (1963), 'Combustion noise', J. Inst. Fuel 36, 12-16.

Clavin, P. and Siggia, E. D. (1991), 'Turbulent premixed flames and sound generation', Combust. Sci. Technol. 78, 147-155.

Crighton, D. G., Dowling, A. P., Ffowcs Williams, J. E., Heckl, M. and Leppington, F. G. (1992), Modern Methods in Analytical Acoustics: Lecture Notes, Springer-Verlag, London.

Depuru Mohan, N., Dowling, A., Karabasov, S., Xia, H., Graham, O., Hynes, T. and Tucker, P. (2015), 'Acoustic sources and far-field noise of chevron and round jets', AIAA J. (5), 1-16.

Domingo, P., Vervisch, L., Payet, S. and Hauguel, R. (2005), 'DNS of a premixed turbulent $\mathrm{V}$ flame and LES of a ducted flame using a FSD-PDF subgrid scale closure with FPI-tabulated chemistry', Combust. Flame 143, 566-586.

Dowling, A. P. (1979), Mean temperature and flow effects on combustion noise, AIAA Paper 1979-0590.

Dowling, A. P. and Ffowcs Williams, J. E. (1983), Sound and Sources of Sound, Ellis Horwood Ltd., Chichester, England.

Dunstan, T. D., Swaminathan, N., Bray, K. N. C. and Cant, R. S. (2011), 'Geometrical properties and turbulent flame speed measurements in stationary premixed $\mathrm{V}$-flames using direct numerical simulations', Flow Turbul. Combust. 87(2-3), 237-259.

Gouldin, F. C. (1996), Combustion intensity and burning rate integral of premixed flames, in 'Twenty-Sixth Symposium (International) on Combustion', pp. 381-388.

Hemchandra, S. and Lieuwen, T. (2010), 'Local consumption speed of turbulent premixed flames: an analysis of 'memory effects", Combust. Flame 257, 1435-1441.

Hirsch, C., Wäsle, J., Winkler, A. and Sattlemayer, T. (2007), 'A spectral model for the sound pressure from turbulent premixed combustion', Proc. Combust. Inst. 31, 1435-1441. 
Hurle, I. R., Price, R. B., Sugden, T. M. and Thomas, A. (1968), 'Sound emission from open turbulent premixed flames', Proc. R. Soc. Lond. A 303, 409-427.

Jones, H. (1979), 'The generation of sound by flames', Proc. R. Soc. Lond. A 367, 291-309.

Karabasov, S. A., Afsar, M. Z., Hynes, T. P., Dowling, A. P., McMullan, W. A., Pokora, C. D., Page, G. J. and McGuirk, J. J. (2010), 'Jet noise: Acoustic analogy informed by large eddy simulation', AIAA J. 48(7), 1312-1325.

Karabasov, S. A., Bogey, C. and Hynes, T. (2011), Computation of the noise of initially laminar jets using a statistical approach for the acoustic analogy: application and discussion, AIAA Paper 2011-2929.

Kilham, J. H. and Kirmani, N. (1979), 'The effect of turbulence on premixed flame noise', Proc. Combust. Inst. 17, 327-336.

Kotake, S. (1975), 'On combustion noise related to chemical relations', J. Sound Vib. 42(3), 399-410.

Kotake, S. and Takamoto, K. (1987), 'Combustion noise: Effects of the shape and size of burner nozzle', J. Sound Vib. 112(2), 345-354.

Kotake, S. and Takamoto, K. (1990), 'Combustion noise: Effects of the velocity turbulence of unburned mixture', J. Sound Vib. 139(1), 9-20.

Lighthill, M. J. (1952), 'On sound generated aerodynamically. I. General theory', Proc. R. Soc. Lond. A 211, 564-587.

Lighthill, M. J. (1954), 'On sound generated aerodynamically. II. Turbulence as a source of sound', Proc. R. Soc. Lond. A 222, 1-32.

Lipatnikov, A. N. and Chomaik, J. (2002), 'Turbulent flame speed and thickness: phenomenology, evaluation, and application in multi-dimensional simulations', Prog. Energy Combust. Sci. 28, 1-74.

Liu, Y., Dowling, A. P., Swaminathan, N., Dunstan, T. D., Morvant, R., Macquisten, M. A. and Caracciolo, L. (2011), Prediction of noise source for an aeroengine combustor, AIAA Paper 2011-2913.

Liu, Y., Dowling, A. P., Swaminathan, N. and Dunstan, T. D. (2012), 'Spatial correlation of heat release rate and sound emission from turbulent premixed flames', Combust. Flame 159(7), 2430-2440.

Liu, Y., Dowling, A. P., Dunstan, T. D. and Swaminathan, N. (2012a), Modeling of combustion noise spectrum from turbulent premixed flames, in 'Acoustics 2012', number 00734, Nantes, France.

Liu, Y., Dowling, A. P., Swaminathan, N., Morvant, R., Macquisten, M. A. and Caracciolo, L. (2014), 'Prediction of combustion noise for an aeroengine combustor', J. Propul. Power 30(1), 114-122. 
Liu, Y. (2015), 'Two-time correlation of heat release rate and spectrum of combustion noise from turbulent premixed flames', J. Sound Vib. 353, 119-134.

Liu, Y. and Echekki, T. (2015), Modelling of combustion noise spectrum using temporal correlations of heat release rate from turbulent premixed flames, AIAA Paper 2015-2970.

Nada, Y., Shiwaku, N., Tanahashi., M. and Miyauchi, T. (2005), Fractal characteristics of hydrogen-air turbulent premixed flames, in "5th Asia-Pacific Conference on Combustion, 2005', The University of Adelaide, Australia.

Nada, Y., Tanahashi., M. and Miyauchi, T. (2004), 'Effect of turbulence characteristics on local flame structure of $\mathrm{H}_{2}$-air premixed flames', J. Turbul. 5(1), 1-15.

Poinsot, T. J. and Lele, S. K. (1992), 'Boundary conditions for direct simulations of compressible viscous flows', J. Comp. Phys. 101, 104-129.

Poinsot, T. J. and Veynante, D. (2005), Theoretical and Numerical Combustion, 2nd edn, Edwards Inc., Philadelphia, PA, USA, chapter 1-2.

Putnam, A. A. (1976), 'Combustion roar of seven industrial gas burners', J. Inst. Fuel 49, 135-138.

Rajaram, R. (2007), Characteristics of sound radiation from turbulent premixed flames, PhD thesis, Department of Aerospace Engineering, Georgia Institute of Technology, Atlanta, Georgia, USA.

Rajaram, R. and Lieuwen, T. (2003), 'Parametric studies of acoustic radiation from turbulent premixed flames', Combust. Sci. Technol. 175, 2269-2298.

Rajaram, R. and Lieuwen, T. (2009), 'Acoustic radiation from turbulent premixed flames', J. Fluid Mech. 637, 357-385.

Roberts, J. P. and Leventhall, H. G. (1973), 'Noise sources in turbulent gaseous premixed flames', Appl. Acoust. 6(301-308).

Rutland, C. J. and Cant, R. S. (1994), Turbulent transport in premixed flames, Proceedings of Summer Program, Centre for Turbulence Research, NASA Ames/Stanford University, California, USA.

Sattler, S. S., Knaus, D. A. and Gouldin, F. C. (2002), 'Determination of three-dimensional flamelet orientation distributions in turbulent V-flames from two-dimensional image data', Proc. Combust. Inst. 29, 1785-1792.

Shepherd, I. G. (1996), Flame surface density and burning rate in premixed turbulent flames, in 'Twenty-Sixth Symposium (International) on Combustion', pp. 373-379.

Smith, K. O. and Gouldin, F. C. (1978), 'Experimental investigation of flow turbulence effects on premixed methane-air flames', Progr. Astronaut. Aero. 58, 37-54.

Strahle, W. C. (1971), 'On combustion generated noise', J. Fluid Mech. 49, 399-414. 
Strahle, W. C. (1973), 'Refraction, convection and diffusion flame effects in combustion generated noise', Proc. Combust. Inst. 13, 527-535.

Strahle, W. C. (1978), 'Combustion noise', Prog. Energy Combust. Sci. 4, 157-176.

Strahle, W. C. and Shivashankara, B. N. (1975), 'A rational correlation of combustion noise results from open turbulent premixed flames', Proc. Combust. Inst. 15, 1379-1385.

Swaminathan, N., Balachandran, R., Xu, G. and Dowling, A. P. (2011), 'On the correlation of heat release rate in turbulent premixed flames', Proc. Combust. Inst. 33, 1533-1541.

Swaminathan, N., Xu, G., Dowling, A. P. and Balachandran, R. (2011a), 'Heat release rate correlation and combustion noise in premixed flames', J. Fluid Mech. 681, 80-115.

Talei, M., Brear, M. J. and Hawkes, E. R. (2011), 'Sound generation by laminar premixed flame annihilation', J. Fluid Mech. 679, 194-218.

Talei, M., Brear, M. J. and Hawkes, E. R. (2012), 'A parametric study of sound generation by premixed laminar flame annihilation', Combust. Flame 159(2), 757-769.

Talei, M., Hawkes, E. R. and Brear, M. J. (2013), 'A direct numerical simulation study of frequency and lewis number effects on sound generation by two-dimensional forced laminar premixed flames', Proc. Combust. Inst. 34(1), 1093-1100.

Tam, C. K. W. (2015), On the spectrum of combustion noise, AIAA Paper 2015-2969.

Veynante, D., Duclos, J. M. and Piana, J. (1994), Experimental analysis of flamelet models for premixed turbulent combustion, in 'Twenty-Fifth Symposium (International) on Combustion', pp. 1249-1256.

Wäsle, J., Winkler, A. and Sattlemayer, T. (2005), 'Spatial coherence of the heat release fluctuations in turbulent jet and swirl flames', Flow Turbul. Combust. 75(1-4), 29-50. 